\title{
Taxonomy of tribe Neillieae (Rosaceae): Neillia
}

\author{
Sang-Hun Oh* \\ Department of Biology, Daejeon University, Daejeon 34520, Korea \\ (Received 16 February 2016; Revised 6 March 2016; Accepted 6 March 2016)

\section{나도국수나무족(장미과)의 분류: 나도국수나무속} \\ 오상훈* \\ 대전대학교 생명과학과
}

\begin{abstract}
Neillieae was traditionally recognized as a small tribe in Rosaceae, which consists of Neillia, Stephanan$d r a$, and Physocarpus. Recently, Stephanandra was merged into Neillia based on molecular phylogenetic analyses, meaning that Neillieae now contains Neillia and Physocarpus. The genus Neillia is distinguished from Physocarpus by ovate to lanceolate leaves with acuminate to caudate apices, racemose or paniculate inflorescences, and a unicarpellate (rarely bicarpellate) gynoecium. Plants of Neillia are distributed from the Himalayas across China and Korea to Japan in the east, and south to Indonesia. This study provides a taxonomic treatment of Neillia based on a morphological examination of herbarium specimens, including types, and field observations, as the second part of the taxonomic revision of the tribe Neillieae. A summary of the phylogeny of Neillia, keys to the species, nomenclatural reviews, descriptions, distribution maps, and lists of specimens examined are provided. Twelve species with ten varieties are recognized in Neillia. A lectotype was designated here for the following species: $N$. sinensis f. glanduligera and $N$. thyrsiflora.
\end{abstract}

Keywords: Neillieae, Neillia, revision

적 요: 나도국수나무족(Neillieae)은 전통적으로 나도국수나무속(Neillia), 국수나무속(Stephanandra), 산국수나 무속(Physocarpus)의 3속으로 구성된 장미과의 분류군으로 인식되어 왔다. 그러나, 국수나무속은 최근의 분자계 통학적 연구에 근거하여 나도국수나무속에 포함되어, 나도국수나무족은 나도국수나무속과 산국수나무속의 2속 으로 구성된 족으로 인식된다. 나도국수나무속은 난형 내지 피침형의 잎, 점첨두 내지 꼬리형의 엽선, 총상 또 는 원추화서 및 단심피의 암술을 갖는 특징에 의해 산국수나무속과 뚜렷이 구분된다. 본 속의 식물은 히말라야 에서 중국 및 한국을 거쳐 동쪽으로 일본, 남쪽으로 인도네시아에 걸쳐 분포한다. 본 연구에서는 나도국수나무 족의 종속지적 연구의 일환으로 나도국수나무속의 분류를 제시하였다. 기준표본을 포함한 표본 조사 및 야외 조 사를 바탕으로 형태형질 분석 결과와 현재까지 밝혀진 분자계통학적 연구 결과를 토대로 각 종에 대한 검색표, 모든 학명에 대한 자세한 명명법적인 검증 및 처리를 포함하여 나도국수나무속 및 각 종에 대한 기재, 분포도 및 조사한 표본의 목록을 제시하였다. 본 연구에서는 나도국수나무속에 12 종을 인식하였으며, 이 중 3 종에서 총 10 변종을 인식하였다. 또한, N. sinensis f. glanduligera 및 N. thyrsiffora의 학명에 대한 선정기준표본을 지정하였다.

주요어: 나도국수나무족, 나도국수나무, 종속지

Tribe Neillieae (Rosaceae) consists of Neillia D. Don, including Stephanandra Siebold \& Zucc., and Physocarpus
(Cambess.) Raf. (Maximowicz, 1879; Schulze-Menz, 1964; Oh, 2006). In the previous part of the taxonomic revision of

\footnotetext{
*Author for correspondence: soh42@dju.kr
} 
tribe Neillieae (Oh, 2015), the nomenclatural history of tribe Neillieae and the taxonomic treatment of Physocarpus were provided. This study provides a taxonomic treatment of Neillia, including Stephanandra.

In the traditional sense, Neillia was recognized as having nine species of deciduous shrubs that are distributed from the Himalayas across China to Korea in the east, and south to Indonesia (Vidal, 1963; Schulze-Menz, 1964; Cullen, 1971; Kalkman, 1993). Stephanandra was considered as a small genus consisting of three species indigenous to China, Taiwan, Korea, and Japan (Ohwi, 1965; Yu and Ku, 1974; Lee, 1980). Morphological characters of the fruits and seeds, such as the degree of dehiscence of the follicles, the orientation of the style, the number of seeds per follicle, and the texture of the seed coat, have been used to distinguish Neillia from Stephanandra (Vidal, 1963; Cullen, 1971). Oh (2002, 2006), however, determined that these characters do not clearly separate the two genera. The hypanthium shape and capitate-glandular trichomes on the surface of the hypanthium are useful to differentiate the two genera (Oh, 2002, 2006). Members of Neillia in the traditional sense have campanulate to cylindric hypanthia with capitate-glandular trichomes developed after anthesis, while species of Stephanandra have cupulate hypanthia without capitate-glandular trichomes.

Phylogenetic analyses using DNA sequences of chloroplast and nuclear genes have suggested that Neillia and Stephanandra together form a strongly supported monophyletic group (Oh and Potter, 2003, 2005; Oh, 2013). However, none of the molecular data support the reciprocal monophyly of Neillia and Stephanandra. Specifically, DNA sequence data of the chloroplast trnL-trnF, trnD-trnT, matK-trnK, and psbA-trnK regions and the second intron of $L E A F Y$ congruently support the contention that Stephanandra is monophyletic and nested within Neillia, making Neillia a paraphyletic genus (Fig. 1A, B; Oh and Potter, 2003, 2005). Internal transcribed spacer (ITS) and external transcribed spacer (ETS) data did not support the monophyly of Stephanandra, placing S. tanakae as a sister to the weakly supported clade of Neillia, S. incisa, and $S$. chinensis, but neither Stephanandra nor Neillia was supported as monophyletic (Fig. 1C; Oh and Potter, 2003, 2005). In the nuclear PI data, neither Neillia nor Stephanandra was supported as monophyletic (Fig. 1D; Oh, 2013).

Monophyly of Neillia plus Stephanandra has also been strongly supported in the broad analyses of Rosaceae (Morgan et al., 1994; Potter et al., 2002; Potter et al., 2007). The close relationship between Neillia and Stephanandra is supported by several morphological characteristics; species in both genera have ovate to lanceolate leaves with acuminate to caudate apices, racemose or paniculate inflorescences, and a single (rarely two)
A. cPDNA

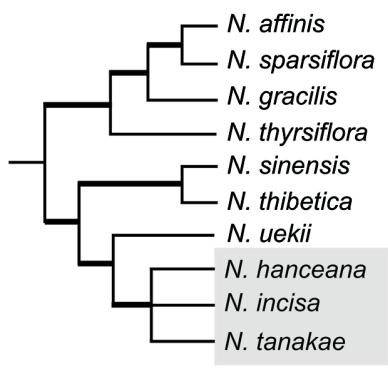

C. rDNA

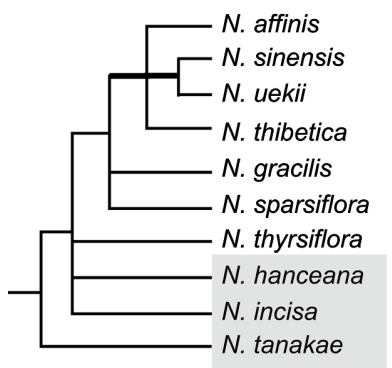

B. LEAFY

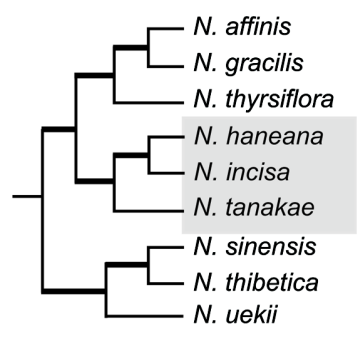

D. PISTILLATA

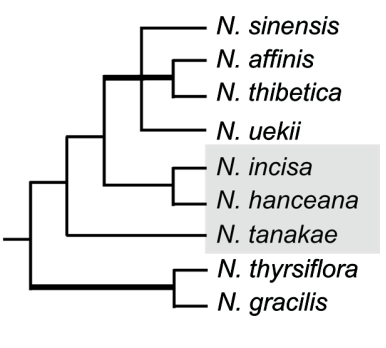

Fig. 1. Phylogenetic summary trees of Neillia based on different data sets. A thick line indicates strongly supported clade with greater than $70 \%$ of bootstrap values in MP and/or ML and 0.95 of posterior probability in Bayesian analysis. Species of Stephanandra were marked with a shaded box. A. Combined chloroplast regions of trnL-trnF, trnD-trnT, matK-trnK, and psbA-trnK (Oh and Potter, 2003,2005 ). B. The second intron of $L E A F Y$ (Oh and Potter, 2003, 2005). C. Combined spacer regions of nrDNA ITS and ETS (Oh and Potter, 2003, 2005). D. PISTILLATA data (Oh, 2013).

carpel per flower. Oh (2006) combined Stephanandra into Neillia following the recommendation of Oh and Potter (2005). The genus Neillia can be distinguished from Physocarpus by ovate to lanceolate leaves with acuminate to caudate apices, racemose or paniculate inflorescences, and a unicarpellate (rarely bicarpellate) gynoecium.

In a series of two papers, a comprehensive taxonomy of tribe Neillieae reflecting most recent morphological and molecular analysis of the group (Oh and Potter, 2003, 2005; Oh, 2004, 2006, 2015; Oh et al., 2010) is provided. As the second part of the taxonomic revision of tribe Neillieae, this paper provides a morphological description of Neillia along with a detailed synonymy and a key to the species.

\section{Materials and Methods}

Approximately 2,000 herbarium specimens of Neillia were examined. Specimens were borrowed from major herbaria, including A, BM, E, GH, K, KPM, L, MO, NY, P, PE, and UC (Thiers, 2016). Specimens at DAV, KB, and KUN, and those collected by the author were also examined. The data 
presented here, including plant measurements and distributions, were obtained mainly from herbarium specimens. Where more than 100 herbarium specimens were studied for a taxon, a subset of the total specimens examined is cited.

\section{Taxonomic Treatment}

Neillieae Maxim., Trudy Imp. S.-Peterburgsk. Bot. Sada 6: 216. 1879.-TYPE: Neillia D. Don

Description of Neillieae and taxonomic treatment of Physocarpus can be found in Oh (2015).

\section{Key to the genera of tribe Neillieae}

1. Leaves covered with stellate trichomes of various density; inflorescences corymbs; follicles dehiscent along both ventral and dorsal sutures Physocarpus

1. Leaves covered with simple trichomes of various density; inflorescences racemes or panicles of racemes; follicles dehiscent along ventral suture only

Neillia (including Stephanandra)

Neillia D. Don, Prodr. Fl. Nepal 228, 1825.-TYPE: Neillia thyrsiflora D. Don.

Adenilema Blume, Bijdr. 1120, 1826.-TYPE: Adenilema fallax Blume.

Stephanandra Siebold \& Zucc., Abh. Math.-Phys. Cl. Königl. Bayer. Akad. Wiss. 3: 740, 1843.-TYPE: Stephanandra flexuosa Siebold \& Zucc. [=Stephanandra incisa (Thunb.) Zabel].

Neilliaceae Miq., Fl. Ned. Ind. 1: 390, 1855.-TYPE: Neillia D. Don.

Shrubs with erect, spreading, scandent, or decumbent branches; pubescence usually present, the trichomes generally simple, unicellular, capitate-glandular, or rarely stellate. Leaves on the flowering branches with or without multiple, superposed buds in their axils; blade ovate to lanceolate, palmately 3-lobed, the margins doubly serrated; petioles evident; stipules linear, lanceolate, or ovate, deciduous or persistent. Inflorescences terminating lateral branches of the season, bracteate, racemes or panicles of racemes; flowers fascicled at the apex of the inflorescence or more or less evenly distributed along the inflorescence rachis. Hypanthium cupulate, campanulate to cylindric; sepals spreading when flowering becoming erect after anthesis, ovate or triangular; petals spreading, orbicular, white or pale pink; stamens 10-30, exserted or not, arising from the rim of hypanthium, some persistent; carpels 1 , occasionally 2 , rarely $3-5$, free or weakly connate at the base, glabrous to densely pubescent, the style terminal; ovules $2-10$ per carpel. Fruit a follicle, not inflated, dehiscent along ventral suture; seeds
1-10 per follicle, yellowish brown or dark brown. Chromosome number: $2 n=18$.

\section{Key to the species of Neillia}

1. Hypanthia cupulate, without capitate-glandular trichomes on the surface at the fruiting stage.

2. Stamens 15-20; sepals apiculate at the apex

9. N. tanakae

2. Stamens 10; sepals obtuse to rounded at the apex.

3. Leaves deeply 3-5-lobed; the middle lobes with 35 pairs of secondary veins …………..... 4. N. incisa

3. Leaves shallowly 3-lobed; the middle lobes with $>5$ pairs of secondary veins ………..... 3. $\mathrm{N}$. hanceana

1. Hypanthia campanulate to cylindric, with capitate-glandular trichomes on the surface at the fruiting stage.

4. Shrubs up to $0.5 \mathrm{~m}$ high, with decumbent branches …. 2. $N$. gracilis

4. Shrubs $>1 \mathrm{~m}$ high, with erect, spreading, or scandent branches.

5. Flowering branches and petioles with capitateglandular trichomes ………............ 8. N. sparsiflora

5. Flowering branches and petioles without capitateglandular trichomes.

6. Hypanthia cylindric or cylindric-campanulate.

7. Inflorescences with 10-20 flowers; pedicels 2.2-7 mm long; hypanthia 6-9 $\mathrm{mm}$ long ......... 7. $N$. sinensis

7. Inflorescences with 20-60 flowers; pedicels 0.6-2 mm long; hypanthia 4-5.5 mm long ….

10. N. thibetica

6. Hypanthia campanulate or globular-campanulate.

8. Leaves on the flowering branches with multiple, superposed buds in their axils.

9. Pedicels $0.5-1.4 \mathrm{~cm}$ long; ovules $8-10$ per carpel …....................... 11. N. thyrsiflora

9. Pedicels 2.5-5 cm long; ovules 3-4 per carpel ………………... 6. $N$. serratisepala

8. Leaves on the flowering branches without multiple, superposed buds in their axils.

10. Inflorescence rachis with stellate 12. N. uekii

10. Inflorescence rachis with simple unicellular trichomes; Bhutan, China, Nepal.

11. Petals pale pink; carpels $1-5$, densely pubescent throughout the surface …. 1. N. affinis

11. Petals white; carpel 1 , glabrous …….. 5. N. rubiflora 
1. Neillia affinis Hemsl., J. Linn. Soc., Bot. 29: 304, 1892.TYPE: China. Sichuan: without specific locality, Feb 1890, Henry 8968 (lectotype: K!, designated by Vidal, Adansonia 3: 156, 1963; isolectotypes: GH!, P!). Syntype: CHINA. Sichuan: Neighborhood of Tachienlu, elev. 9,000-13,500 ft., Pratt 347 (K!).

Shrubs to $2 \mathrm{~m}$ high, with erect, spreading, or scandent branches. Leaves on the flowering branches without multiple, superposed buds in their axils; blades ovate to lanceolate, 3$6(-10) \mathrm{cm}$ long, 2.1-5.5 (-10) cm wide, shallowly 3-lobed, the apex acute to caudate, the base cordate to rounded, the margin doubly serrate, the upper and lower surfaces glabrous to moderately pubescent; petioles $0.8-2 \mathrm{~cm}$ long; stipules ovate to lanceolate, 5-10 $\mathrm{mm}$ long, serrate or entire, deciduous after anthesis. Inflorescences racemes or panicles of racemes, if panicles, 4-5 branched, 3-6 cm long, densely pubescent, more than 10-flowered; flowers fascicled at the apex of the inflorescence, or more or less evenly distributed along the inflorescence rachis; bracts lanceolate, 5-6 mm long; pedicels 1-4 mm long. Hypanthium globular-campanulate to campanulate, $3-5 \mathrm{~mm}$ long, $3.8-4.8 \mathrm{~mm}$ wide at the widest point, densely pubescent, capitate-glandular trichomes developing after anthesis; sepals triangular, 2.5-7 mm long, acuminate at the apex, pubescent on both surfaces; petals orbicular, pale pink; stamens 15-25, the longest filaments ca. $1.8 \mathrm{~mm}$ long; carpels $1-5$, if more than 1 , free or weakly connate at the base, densely pubescent throughout the surface; ovules 6-8 per carpel. Follicles ca. $4 \mathrm{~mm}$ long, with more or less erect beaks; seeds 6 per follicle, ca. $2 \mathrm{~mm}$ long, dark brown.

Neillia affinis is similar to N. rubiflora in having densely pubescent globular-campanulate hypanthia. Both species can have racemes and panicles, although racemes are more common in $N$. affinis and panicles are common in N. rubiflora. Neillia affinis can be distinguished from $N$. rubiflora by its densely pubescent carpels. Four varieties may be distinguished as follows:

1. Carpels 3-5, free ……………………........ var. polygyna

1. Carpels 1-2, free or weakly connate at the base.

2. Inflorescences racemes, 5-10 flowers; flowers fascicled at the apex of the inflorescence …….... var. pauciflora

2. Inflorescences racemes or panicles of racemes, $>10$ flowers; flowers more or less evenly distributed along the inflorescence rachis.

3. Sepals as long as or longer than the hypanthium, 6$7 \mathrm{~mm}$ long var. longisepala

3. Sepals shorter than the hypanthium, 2.5-5 mm long

\section{1a. Neillia affinis var. affinis}

Shrubs to $2 \mathrm{~m}$ high, with erect, spreading, or scandent branches. Blades ovate, 3-6 (-10) cm long, 2.8-5.5 (-10) cm wide, the apex acute to acuminate, the base cordate to rounded, the upper and lower surfaces glabrous to sparsely pubescent. Inflorescences racemes or panicles of racemes, if panicles, 45 branched, densely pubescent, 10-many-flowered; flowers more or less evenly distributed along the inflorescence rachis. Carpels 1-2, if 2, weakly connate at least at the base.

Distribution. China in Sichuan and Yunnan (Fig. 2); recorded by Cullen (1971) from Myanmar; 1,400-3,200 m.

Plants of this variety often have two carpels that are occasionally connate at the base as in Physocarpus.

Specimens examined. CHINA. Sichuan: without specific locality, elev. 8,000 ft., Jul 1904, Wilson 3560 (A, P); Opien Hsien, 14 Jul 1930, Fang 4694 (A, NY); Nanchuan Hsien, elev. 8,000-9,000 ft., 25 May 1928, Fang 935 (A); Nanchuan-Xian, elev. 8,000-9,000 ft., 25 May 1928, Feng 935 (A); Hsian-hsiangling, elev. 2,000 m, 21 May 1922, Smith 1865 (A); Ma-ping Hsien, elev. 2,400 m, 27 May 1931, Wang 22992 (A); cliffs of Mt. Wu-shan, elev. 8,000 ft., Jul 1903, Wilson 3559 (A-2 sheets); Mt. Wa-shan, Jun and Sep 1908, Wilson $916 a$ (A-3 sheets); Jueishe Hsien, elev. 2,500 m, 31 May 1932, Yu 941 (A). Yunnan: without specific locality and date, Forrest 7584 (A); inter fluvios Landsang-djiang (Mekong) et Lu-djiang (Salween), ca. $28^{\circ} \mathrm{N}$, in valle a jugo Si-la, a vicum Tsekuo descendente, elev. 2,3002,500 m, 15 Jun 1916, Handel-Mazzetti 8884 (A); Fuchuan range, $\mathrm{W}$ of the Mekopng-Salween divide and $\mathrm{W}$ of Wei-hsi, elev. 10,500 ft., Aug-Sep 1932, Rock 23275 (A, NY); Fuchuan range, $\mathrm{W}$ of the Mekong-Salween divide and $\mathrm{W}$ of Wei-hsi, elev. 10,500 ft., May-Jun 1932, Rock 22729 (A, NY); Yi-liang Hsien, elev. 1,400 m, 27 May 1932, Tsai 52055 (A); Yong-shan Hsien,

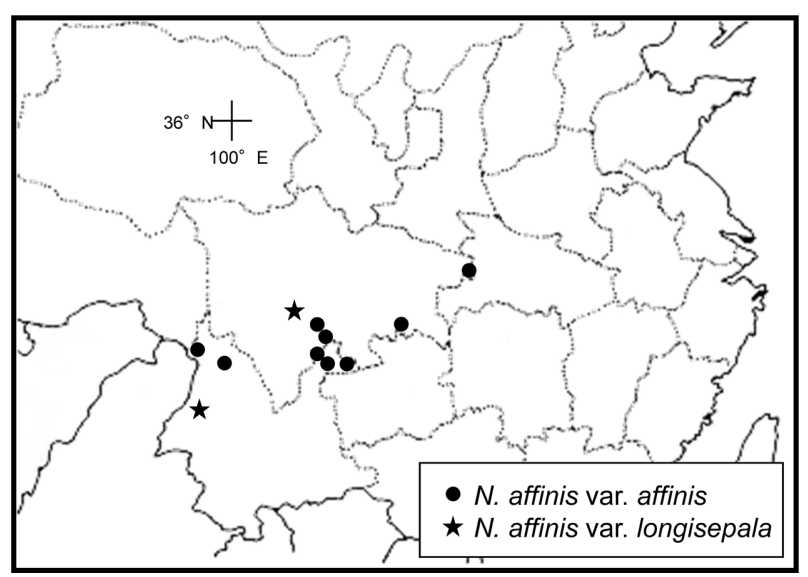

Fig. 2. The distributions of $N$. affinis var. affinis and var. longisepala. 
elev. 2,100 m, 31 May 1932, Tsai 50949 (A); Wei-hsi Hsien, Yeh-Chih, elev. 3,200 m, Aug 1935, Wang 68154 (A); Chenkang, Snow range, elev. 2,500 m, 31 Jul 1938, Yu 17115 (A); Kengma, Chuichayko, elev. 2,500 m, 19 Aug 1938, Yu 17297 (A).

1b. Neillia affinis var. longisepala Cullen, J. Arnold Arbor. 52: 147, 1971.-TYPE: China. Yunnan: Shweli-Salween divide, $25^{\circ} 20^{\prime} \mathrm{N}$, elev. 3,000 m, shrub of 4-5 ft, flowers creamy yellow, in scrub and thickets in side valleys, Aug 1918. Forrest 17605 (holotype: E!; isotypes: A!, WSY).

Shrubs to $2 \mathrm{~m}$ high, with erect or spreading branches. Blades ovate to lanceolate, $3-6 \mathrm{~cm}$ long, $3-5.5 \mathrm{~cm}$ wide, the apex acuminate to caudate, the base cordate, the upper and lower surfaces glabrous to sparsely pubescent. Inflorescences racemes, densely pubescent, more than 10-flowered; flowers more or less evenly distributed along the inflorescence rachis. Carpel 1.

Distribution. China in Sichuan and Yunnan (Fig. 2).

This variety is characterized by its ovate to lanceolate leaves and long caudate sepals.

Specimens examined. CHINA. Sichuan: Mt. Omei, Jun 1904, Wilson 4886 (A).

1c. Neillia affinis var. pauciflora (Rehder) J. E. Vidal, Adansonia 3: 156, 1963; Neillia pauciflora Rehder in Sargent, Pl. Wilson. 1: 437, 1913.-TYPE: China. Yunnan: Mengtze, N. Mt., elev. 7,000 ft., Henry 10231 (lectotype: K!, designated by Vidal, Adansonia 3: 156, 1963; isolectotypes: A-2 sheets!, NY3 sheets!).

Shrubs to $1.5 \mathrm{~m}$ high, with erect or spreading branches. Blades ovate, $3-5 \mathrm{~cm}$ long, $2.1-4 \mathrm{~cm}$ wide, the apex acute to

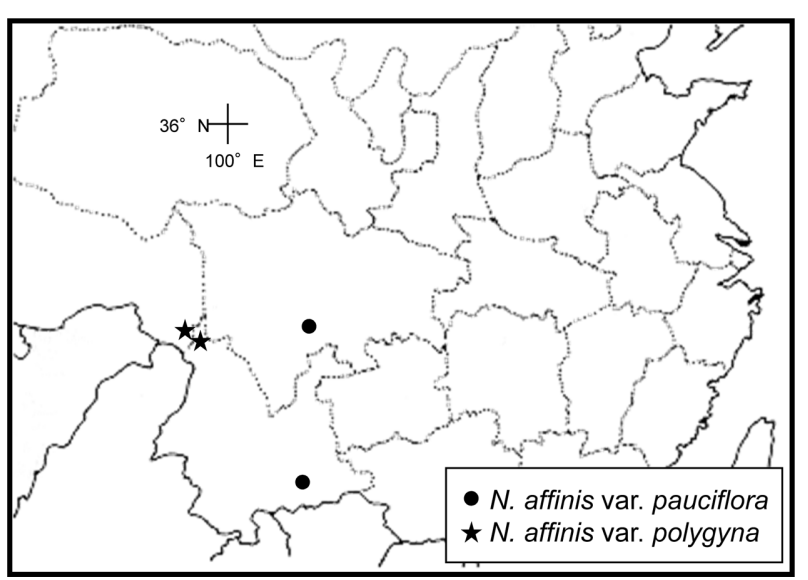

Fig. 3. The distributions of $N$. affinis var. paciflora and var. polygyna. acuminate, the base cordate, the upper and lower surfaces moderately pubescent; stipules ovate, entire. Inflorescences racemes, 2-3 cm long, densely pubescent, 5-10-flowered; flowers fascicled at the apex of the inflorescence; bracts lanceolate, 5-6 mm long; pedicels 1.5-2 mm long. Carpels 1-2.

Distribution. China in Sichuan and Yunnan (Fig. 3); 2,0002,300 m.

This variety can be distinguished from the other varieties by having flowers fascicled at the apex of the inflorescence and by having fewer flowers per inflorescence.

Typification of $N$. affinis var. pauciflora is complicated. Rehder (1913) cited two specimens in the original description of $N$. pauciflora, in which he wrote "Nos. 10231a, type, 10231. Therefore, it is unclear whether 10231 or $10231 a$ was cited as the type. I examined original specimens from A, K, and NY and found that one specimen at NY bears both numbers on one specimen with one plant, while others have one or the others of the two numbers. All of the specimens cited for the taxon were collected in the same place and on the same day by the same person, and perfectly match. Vidal (1963) cited Henry $10231 a$ at $\mathrm{K}$ as the type, but Henry's specimen at $\mathrm{K}$ bears number 10231. Here, I correct the collection number of the lectotype designated by Vidal at K as number 10231 .

Specimens examined. CHINA. Sichuan: Mt. Omei, W Sichuan, elev. 2,300 m, 26 Jul 1939, Sun \& Chang 1234 (A); Mt. Omei, Yen-wang-po, elev. 2,400 m, 18 Aug 1940, Tu 272 (A). Yunnan: Environs de Yunnan-sen, 22 may 1899, Ducloux 630 (P); Hay Y, près Lou lan, 1 May 1907, Ducloux 5214 (P); hills to the E of Tengyueh, $25^{\circ} \mathrm{N}$, elev. 2,000 m, May 1912, Forrest 7854 (A).

1d. Neillia affinis var. polygyna Cardot ex J. E. Vidal, Adansonia 3: 156, 1963.-TYPE: China. Tibet: "Tsékou," 20 Jun 1895, Soulié 1247 (holotype: P!).

Shrubs to $2 \mathrm{~m}$ high, with erect or spreading branches. Blades ovate, 3-6 (-10) cm long, 2.8-5.5 (-10) cm wide, the apex acute to acuminate, the base cordate to rounded, the upper and lower surfaces glabrous to sparsely pubescent. Inflorescences racemes, densely pubescent, more than 10-flowered; flowers more or less evenly distributed along the inflorescence rachis. Carpels 3-5, free.

Distribution. China in Yunnan and Xizang (Tibet) (Fig. 3); 4,000 m.

Specimens examined. CHINA. Yunnan: on the Kari pass, $28^{\circ} \mathrm{N}$, elev. 4,000 m, Jun 1917, Forrest 14002 (A). Xizang (Tibet): Sarong on Doker La, Mekong-Salween divide, $28^{\circ} 20^{\prime} \mathrm{N}$, without date, Forrest 14342 (A). 
2. Neillia gracilis Franch., Pl. Delavay. 1: 202, 1889.-TYPE: China. Yunnan: in cacuminis montis Tche-tchang, prope Tongtchan, elev. 2,500 m, 1 Jun 1882, Delavay 249 (lectotype: P!, designated by Vidal, Adansonia 3: 157, 1963; isolectotype: A!). Syntypes: CHINA. Yunnan: "in monte Yang-in-Chan supra Lankong," elev. 3,000 m, 7 Jun 1886, Delavay 2338 (NY!, P!) \& 3469 (P!).

Shrubs to $0.5 \mathrm{~m}$ high, with decumbent branches. Leaves on the flowering branches without multiple, superposed buds in their axils; blades ovate to broadly ovate, $2.5-3.5 \mathrm{~cm}$ long, 2$3 \mathrm{~cm}$ wide, shallowly 3-lobed, the apex acute to acuminate, the base cordate, the upper and lower surfaces sparsely pubescent; petioles 1-1.6 cm long; stipules ovate, 4-6 mm long, serrate or entire, persistent or deciduous after anthesis. Inflorescences racemes, 1-1.8 cm long, glabrous, 3-8-flowered; flowers fascicled at the apex of the inflorescence; bracts lanceolate, 3$5.5 \mathrm{~mm}$ long; pedicels $0.8-1 \mathrm{~mm}$ long. Hypanthium campanulate, $3.2-3.5 \mathrm{~mm}$ long, $2.8-3.2 \mathrm{~mm}$ wide at the widest point, moderately pubescent, capitate-glandular trichomes developing after anthesis; sepals ovate, $2-3 \mathrm{~mm}$ long, apiculate at the apex, pubescent on both surfaces; petals orbicular, white or pale pink; stamens $15-20$, the longest filaments ca. $1.5 \mathrm{~mm}$ long; carpel 1 , densely pubescent upper half or at the apex; ovules 2 per carpel. Follicles $4.5 \mathrm{~mm}$ long, with more or less erect beaks; seeds 2 per follicle, ca. $2.4 \mathrm{~mm}$ long, dark brown.

Distribution. China Sichuan and Yunnan (Fig. 4); alpine meadows, mixed coniferous forests; locally common; 2,800$3,100 \mathrm{~m}$.

Neillia gracilis, which occurs in Sichuan and Yunnan Provinces of China, is morphologically unique in Neillia. Compared to other species in the genus, which are manybranched shrubs reaching up to $3 \mathrm{~m}$ high, $N$. gracilis can be easily distinguished by its small size, reaching only $50 \mathrm{~cm}$ in

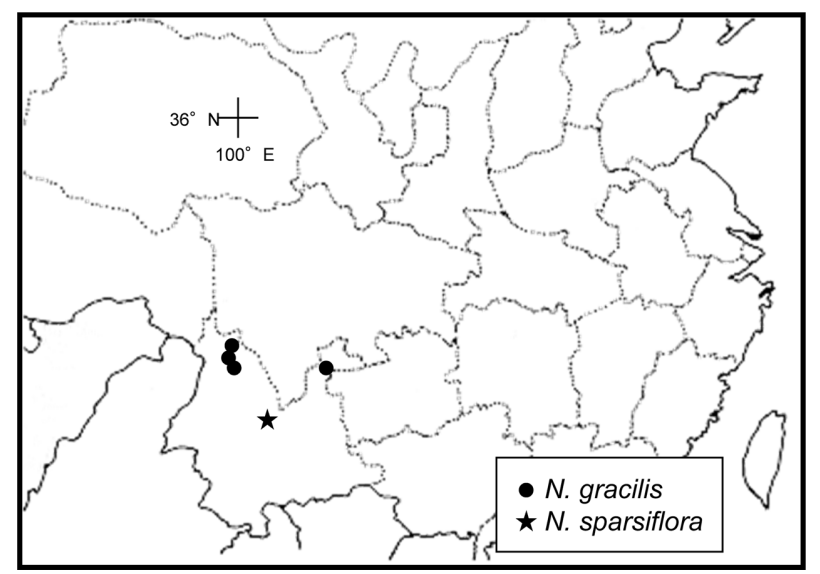

Fig. 4. The distributions of $N$. gracilis and $N$. sparsiflora. height. Plants of this species are suffrutescent and rhizomatous, having only a few decumbent braches with several leaves on each branch. Cullen (1971) considered $N$. gracilis to be closely related to $N$. uekii based on the number of ovules, both having two. However, $N$. gracilis is similar to $N$. affinis in having a broadly ovate blade with three shallow lobes and flowers clustered at the apex of inflorescence.

Specimens examined. CHINA. Sichuan: inter Hunka et Woholo, elev. 3,000 m, 13 Jun 1914, Schneider 1506 (A, GH). Yunnan: NW Likiang, Ah-s-chi, in mixed forests, 25 Jun 1939, Ching 20891 (A); Likiang snow range, in mixed forests, 28 Jun 1939, Ching 30281 (A); Chungtien valley, elev. 3,500 m, 9 Aug 1939, Feng 1936 (A); Lichiang Range, elev. 10,00010,500 ft., $27^{\circ} 12^{\prime}$ N, May 1906, Forrest 2244 (A); Mts S of Likiang, near Hochin and Chiuno, 25-28 May 1922, Rock 4079 (A); Yangtze watershed, prefectural district of Li-kiang, eastern slopes of Likiang snow range, Jun 1922, Rock 4422 (A); Likiang range, 31 Jun 1914, Schneider 2071 (A); N end of Cangshan, Wutaishan, above Huadianba, elev. 3,300 m, 20 May 1981, Sino-British Expedition to Cangshan 0934 (A); LiKiang Hsien, elev. 2,800 m, Jul 1935, Wang 70883 (A).

3. Neillia hanceana (Kuntze) S. H. Oh, Novon 16: 92, 2006; Physocarpus hanceanus Kuntze, Revis. Gen. Pl. 1: 218, 1891, replaced name for Stephanandra chinensis Hance, J. Bot. 20: 210, 1882; Opulaster hanceanus (Kuntze) Kuntze, Revis. Gen. Pl. 2: 949, 1891; Stephanandra flexuosa Siebold \& Zucc. [=Stephanandra incisa (Thunb.) Zabel] var. chinensis (Hance) Pamp., Nuovo Giorn. Bot. Ital. 17: 297, 1910.-TYPE: China. Anhui: "circa urbem Wu-hu," May 1881, Bullock s.n. (holotype: BM).

Shrubs to $3 \mathrm{~m}$ high, with erect or spreading branches. Leaves on the flowering branches without multiple, superposed buds in their axils; blades ovate, $4.3-9 \mathrm{~cm}$ long, $2.7-5.5 \mathrm{~cm}$ wide, shallowly 3-lobed, the apex caudate to acuminate, the base attenuate, cordate, or truncate; the middle lobes with 6-7 pairs of secondary veins, the upper surface glabrous or sparsely pubescent, the lower surface sparsely pubescent; petioles 3$8 \mathrm{~mm}$ long; stipules lanceolate, 7-9 mm long, entire, persistent or deciduous after anthesis. Inflorescences panicles of racemes, 3-8 branched, 6-9 cm long, glabrous; bracts oblanceolate, 2.5$3 \mathrm{~mm}$ long; pedicels 4-5 mm long. Hypanthium cupulate, $1 \mathrm{~mm}$ long, 2-2.2 $\mathrm{mm}$ wide at the rim, glabrous, capitate-glandular trichomes absent; sepals triangular to ovate, $1.3-2 \mathrm{~mm}$ long, obtuse to rounded at the apex, ciliate at the margin; petals obovate, white; stamens 10, the longest filaments ca. $1 \mathrm{~mm}$ long; carpel 1, densely pubescent throughout the surface; ovules 2 
per carpel. Follicles 2-2.4 mm long, with spreading beaks; seeds 1 (rarely 2) per follicle, $1.6-1.9 \mathrm{~mm}$ long, dark brown.

Distribution. China in Anhui, Guangdong, Guizhou, Hubei, Hunan, Jiangxi, and Zhejiang (Fig. 5); locally common; 400$900 \mathrm{~m}$.

The basionym of N. hanceana is Physocarpus hanceanus, a replaced name for Stephanandra chinensis (Kuntze, 1891). When Stephanandra chinensis is transferred to the genus Neillia, the new combination of $N$. chinensis cannot be used because of the existence of $N$. sinensis (Oliver, 1886). The epithets, chinensis and sinensis, are considered as confusingly similar and are treated as homonyms when they are based on different types (ICN Article 53.3; McNeill., 2012). When Kuntze (1891) merged Neillia and Stephanandra into Physocarpus, he simultaneously transferred both Stephanandra chinensis and Neillia sinensis Oliver to the genus Physocarpus. Because of a new combination P. sinensis (Oliver) Kuntze, Physocarpus hanceanus Kuntze is considered a replaced name for Stephanandra chinensis (Oh, 2006).

The leaves of Neillia hanceana are very similar to those of $N$. sinensis, but the two species can be easily distinguished by floral characters. Neillia hanceana has cupulate hypanthia and panicles with white flowers, whereas $N$. sinensis has cylindric hypanthia and racemes with pinkish flowers.

Specimens examined. CHINA. Anhui: Yue Xi Xian, Yaoluoping, elev. 1,100 m, 14 Aug 1997, Xie et al. 97034 (A); Mt. Huang-shan, Wenquan Hot Spring, elev. 700 m, 10 May 1979, Deng \& Yao 79061 (A, NY); Guangdong: Renhwa District, Mt. Danxia-shan, 21-30 May 1936, Tsang 26432 (A); Guizhou: Jiangkou Xian, Heiwan River on the SE side of the Fanjing-shan mountain range in the vicinity of the Ecological Station of the Guizhou Academy of Sciences, elev. 560 m, 24 Aug 1986, Sino-American Guizhou Botanical Expedition 350 (A); Hubei: Mt. Jigong-shan, border of the provinces of Hubei and Hunan, on the divide between the Yangtze and Hwaiho Rivers, elev. 1,500-2,500 ft., 13 Jun 1917, Bailey s.n. (A); Hunnan: Hnlu Xining Xian, in valley, elev. $950 \mathrm{~m}, 26^{\circ} 4^{\prime} \mathrm{N}, 110^{\circ} 8^{\prime} \mathrm{E}, 12$ May 1996, Luo 1355 (A); Jiangxi: Lu-shan Mts., 2 Jul 1983, Chung \& Sun 243 (A, NY); Mt. Dagang-shan, Fenyi city, 28 Aug 1985, Yao 9275 (A, MO, NY); Zhejiang: E Tien-mu, elev. 2,500-3,000 ft., 19 Jun 1927, Hu 1570 (A, UC); Mt. Tienmu-shan, 23 Jul 1930, Liou 92 (NY).

4. Neillia incisa (Thunb.) S. H. Oh, Novon 16: 92, 2006; Spiraea incisa Thunb. in Murray, Syst. Veg. 14: 472. 1784; Stephanandra incisa (Thunb.) Zabel, Gart.-Zeitung (Berlin) 4: 510, 1885.-TYPE: Japan. (holotype: UPS (IDC microfiche!)).
Korean name: Guk-su-na-mu (국수나무)

Stephanandra flexuosa Siebold \& Zucc., Abh. Math.-Phys. Cl. Königl. Bayer. Akad. Wiss. 3: 740, 1843; Physocarpus flexuosus (Siebold \& Zucc.) Kuntze, Revis. Gen. Pl. 1: 219, 1891; Opulaster flexuosus (Siebold \& Zucc.) Kuntze, Revis. Gen. Pl. 2: 949, 1891.-TYPE: Japan. Siebold (holotype: L?).

Stephanandra quadrifissa Nakai, Bot. Mag. (Tokyo) 40: 170, 1926; Stephanandra incisa var. quadrifissa (Nakai) T. B. Lee, Ill. Fl. Kor. 400, 1980.-TYPE: Korea. Kyeonggi-do: Mt. Suraksan, Chung s.n. (holotype: TI).

Stephanandra incisa var. macrophylla Hid. Takahashi, Bull. Kanagawa Pref. Mus., Nat. Sci. 20: 13, 1991.-TYPE: Japan. Honshu: Izu, Kikurajima Isl, Mt. Oyama, elev. 450 m, Jun 1990, Takahashi 77098 (holotype: KPM; isotype: KPM!).

Shrubs to $2.5 \mathrm{~m}$ high, with erect or spreading branches. Leaves on the flowering branches without multiple, superposed buds in their axils; blades ovate to deltoid, 2-5.4 cm long, 1.5$3.8 \mathrm{~cm}$ wide, deeply $3-5$-lobed, the apex caudate to acuminate, the base truncate to cordate; the upper surface glabrous to sparsely pubescent, the lower surface sparsely pubescent; the middle lobes with 3-5 pairs of secondary veins; petioles 4$8 \mathrm{~mm}$ long; stipules lanceolate to linear, 5-7 mm long, entire, deciduous after anthesis. Inflorescences panicles of racemes, 2-6 branched, 2-3 cm long, sparsely pubescent or glabrous; bracts oblanceolate, $0.9-1.6 \mathrm{~mm}$ long; pedicels $2.5-3.5 \mathrm{~mm}$ long. Hypanthium cupulate, 1-1.2 mm long, 2-2.5 mm wide at the rim, glabrous, capitate-glandular trichomes absent; sepals triangular to ovate, $1.1-1.3 \mathrm{~mm}$ long, obtuse to rounded at the apex, ciliate at the margin at the margin; petals obovate, white;

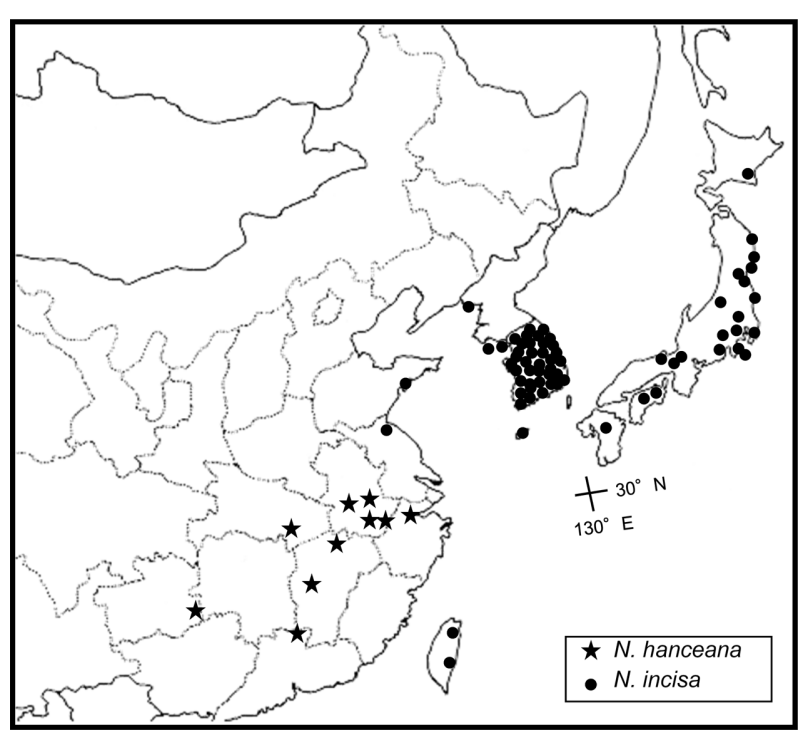

Fig. 5. The distributions of $N$. hanceana and $N$. incisa. 
stamens 10, the longest filaments ca. $1.2 \mathrm{~mm}$ long; carpel 1 , densely pubescent throughout the surface; ovules 2 per carpel. Follicles $1.8-3 \mathrm{~mm}$ long, with spreading beaks; seeds 1 , rarely 2 per follicle, ca. $2 \mathrm{~mm}$ long, dark brown. Chromosome number: $2 n=18$ (Iwatsubo and Naruhashi, 1993).

Distribution. China in Shandong, Taiwan, Korea, and Japan (Fig. 5); mixed deciduous forests; common; 10-1,600 m.

Representative specimens examined. CHINA. Jiangsu: Lianyun District, Lianyungang city, Liuhe (Willow River site), $34^{\circ} 41^{\prime} \mathrm{N}, 119^{\circ} 25^{\prime} \mathrm{E}, 8$ Jun 1987, Sino-American Yuntai Botanical Expedition Team 45003 (A); Shandong: 100 li from Qingdao, Mt. Lao-shan, 1 Jul 1930, Chiao 2644 (A, NY-2 sheets, UC); Taisinggong prope Qingdao, 6 Aug 1936, Licent 13363 (A).

JAPAN. Hokkaido: Hidaka-shicho, Shizunai-cho, ca. 14 km ENE of Shizunai, off Highway 235, mixed decidous streamside forest on tributary of the Shizunai-gawa River, 19 Aug 1977, Wood \& Boufford 3911 (A, MO); Honshu: Chiba Pref., Mt Kiyosumi, Tokyo University forest, Kiyosumi, 25 May 1983, Iketani 712 (A); Fukushima Pref., Iwaki-shi, Mt Yadaijin-yama, 31 Jul 1983, Ohashi et al. 9459 (A); Gumma Pref., Hoshi spa, Niiharu-mura, Tone-gun, 19 Jun 1964, Ono \& Kobayashi 45972 (UC); Kanagawa Pref., Hakone, 7 Jun 1927, Sawada 2101 (A); Hyogo Pref., Mt Nanakusa-yama, Fukuzaki-cho, Kanazaki-gun, 26 May 1979, Tsugaru 5377 (A, NY, MO); Iwate Pref., Shimohei-gun, Yamada-machi, Osawa, elev. ca. 40 m, 9 Jul 1986, Tohda et al. 2017 (A); Kochi Pref., Kamisedo forest road, Siraigawa Hongawa, Tosa-gun, 12 Jul 1995, Takahashi et al. 950712-9 (A); Kyoto Pref., Mt Kibune, about $8 \mathrm{~km} \mathrm{~N}$ of Kyoto city, elev. ca. 450 m, 22 May 1960, Hiroe 13757 (UC); Miyagi Pref., Motoyoshi-gun, Tsuyama-cho, Terakura, on the W side of Okinakura-yama, elev. ca. $130 \mathrm{~m}$, streamside mixed decidous forest, 1 Jun 1990, Boufford \& Wood 25386 (A, NY, MO); Okayama Pref., Sayodami, Tsugawa-mura, 9 May 1951, Nanba s.n. (A); Saitama Pref., Mt. Futagoyama, 7 Jun 1970, Nakaike 15549 (MO); Shiga Pref., Mt Horai in Mts Hira, elev. ca. 800 m, 10 May 1959, Hiroe 13128 (UC); Sizuoka Pref., Ito-shi, Oka, along the road near sea, 4 May 1987, Kawahara 1072 (MO); Tochigi Pref., the border of Kuriyama-mura and Imaichi-shi, elev. ca. 1,025 m, 3 Jul 1984, Ohashi et al. 11061 (A); Tokyo Pref., Asakawa, Hachioji city, 27 Jun 1943, Makino 212467 (MO); Tokyo-do, Hachoijio-shi, Takao-san, Hikage-sawa to the summit, 24 May 1979, Taoda 3721 (A); Yamagata Pref., Yamagata-shi, Kabuto-iwa, 3-4 Aug 1970, Chashi et al. 708066 (MO); Yamanashi Pref., Fujiyoshida-shi, Mt Fuji-san, along Yoshida-guchi climbing path, between Oh'ishi-jaya and Sengen-jinja shrine 20 Jun 1984, Iketani 1560 (A) ; Kyushu: Kumamoto Pref., Takamori-cho, Aso-gun, 7 Jun 1995, Deguchi
8051 (A, MO); Shikoku: Kochi Pref., Kamisedo forest road, Siraigawa Hongawa, Tosa-gun, 12 Jul 1995, Takahashi et al. 950712-9 (A); Tokushima Pref.: Mt Takagi-yama, Kisawamura, Naka-gun, at the edge of Fagus crenata forest near the ridge, elev. 1,500 m, 24 Jul 1986, Murata et al. 56042 (A).

KOREA. Pyeonganbuk-do: French Mine, Taiyudo, 15 Jul 1917, Wilson 8607 (A-2 sheets, MO); Seoul: Mt. Namsan, 5 Jun 1947, Lee s.n. (MO); Mt Inwangsan, N edge of Seoul, $37^{\circ} 35^{\prime} \mathrm{N}, 126^{\circ} 57^{\prime} \mathrm{E}, 22$ May 1955, Moran 5189 (A, UC); Gyeonggi-do: Yongin-si, Cheoin-gu Mohyeon-myeon, Mt. Jeonggwang, elev. 167, 5 Jun 2015, Cho et al. 150605007 (KB); Yeoncheon-gun, Misan-myeon, Gwangdong-ri, Mt. Cheonho, elev. 337, 26 Jul 2010, Jang 367132-0079 (KB); Gwacheon-si, Munwon-dong, Mt. Cheonggye, elev. 124, 2 May 2011, Kim 146 (KB); Gimpo-si, Daegot-myeon, Yagamri, Mt. Seungma, elev. 146, 28 May 2011, Kim et al. Seungma11080 (KB); Suwon-si, Gwonseon-gu, Geumgokdong, Mt. Chilbo, elev. 237, 31 Jul 2012, Kim et al. CB12026 (KB); Paju-si, Paju-eup, Baekseok-ri, Mt. Duri, elev. 50-184, 3 Jun 2003, Kwon \& Kim 791 (KB); Ansan-si, Danwon-gu, Daebunam-dong, elev. 46, 19 May 2012, Lee et al. s.n. (KB); Anyang-si, Manan-gu, Anyang-dong, Mt. Suri, 31 May 2005, Lee et al. s.n. (KB); Gapyeong-gun, Gapyeong-eup, Seunganri, Mt. Yeonin, 26 Apr 2014, Lee s.n. (KB); Mt. Gwanaksan, 6 Jun 1957, Lee s.n. (MO); Gwangneung, 25 Jun 1957, Lee s.n. (MO); Mt. Moraksan, 3 mi SE of Anyang, 15 May 1954, Moran 4271 (GH, MO, UC); Hwaseong-si, Seosin-myeon, Daebudo, elev. 120, 19 May 2009, Lee et al. leecs090202 (KB); Namyangju-si, Byeolnae-myeon, Cheonghak-ri, Mt. Surak, elev. 231, 27 Sep 2012, Lee et al. leecs20121500 (KB); Yangjusi, Yuyang-dong, 30 May 2006, Lee 16 (KB); Uiwang-si, Cheonggye-dong, Mt. Cheonggye, elev. 317, 31 May 2009, Lee BYLee 197 (KB); Mt. Namhansan, 5 Jun 1914, Mills s.n. (UC); Icheon-si, Seolseong-myeon, Daejuk-ri, Mt. Maguk, elev. 264, 18 May 2011, Paik \& Jeong 377142(s0202)-035 (KB); Pocheon-si, Gwanin-myeon, Jung-ri, Jijangbong, elev. 384, 23 Jun 2007, Paik Paik1057 (KB); Yangpyeong-gun, Gaegun-myeon, Gyejeon-ri, Mt. Chueup, elev. 162, 17 Sep 2010, Paik 377111-085 (KB); Yeoju-gun, Gangcheon-myeon, Ganmae-ri, Mt. Bogeum, elev. 111, 23 Jul 2010, Paik 377114051 (KB); Anseong-si, Samjuk-myeon, Naegang-ri, Guksabong, elev. 198, 12 Jun 2011, Yoo \& Han 377143-0132 (KB); Ongjingun, Daeyonpyongdo Island, valley on NW side of island, 17 Aug 1984, Yinger et al. 2463 (A); Gangwon-do: Hwacheon-gun, Sanae-myeon, elev. 635, 8 Jun 2007, Hong et al. K07060806 (KB; Jeongseon-gun, Gohan-eup, Gohan-ri, Mt. Hambaek, elev. 1,321, 24 Jun 2013, Hong et al. HNHM-KB-131013 (KB; Chuncheon-si, Sabuk-myeon, Goseong-ri, Mt. Yonghwa, elev. 
275, 26 Sep 2009, Hwang \& Hong 200907 \& 200920-92 (KB); Goseong-gun, Ganseong-eup, Jinbu-ri, elev. 262, 13 Jun 2008, Hyun et al. s.n. (KB); Cheorwon-gun, Galmal-eup, Dongmak-ri, elev. 217, 20 May 2008, Jeon \& Park ESJeon81742 (KB); Yangyang-gun, Seo-myeon, Galcheon-ri, Guryongnyeong, elev. 1012, 19 Sep 2009, Joung \& Na 1181 (KB); Hoengseong-gun, Gonggeun-myeon, Gagok-ri, Eoreumgol, 15 Sep 2011, Kim kjs100578 (KB); Samcheok-si, Geundeok-myeon, Gungchonri, elev. 173, 7 Jun 2011, Kim \& Jeong SHY2-542 (KB) ;Sokchosi, Jangsa-dong, Youngrangho, elev. 8, 4 Jun 2013, Kim \& Kwak Youngrangho0027 (KB); Yeongwol-gun, Yeongwol-eup, Palgoi-ri, Mt. Taehwa, elev. 332, 29 May 2010, Kim \& Yoon SHY387 (KB); Hongcheon-gun, Nae-myeon, Yuljeon-ri, Saldunsaemteo, elev. 509, 16 Sep 2014, Kim et al. PH140620 (KB); Inje-gun, Girin-myeon, Bangdong-ri, Mt. Bangtae, elev. 587, 30 Jun 2009, Ko HNHM-0254 (KB); Gangneung-si, Gujeong-myeon, Gujeong-ri, Mt. Chilseong, elev. 261, 29 May 2010, Ko \& Son HNHM-2010-1099 (KB); Donghae-si, Samhwa-dong, Mt. Duta, elev. 486, 2 May 2009, Lee et al. 5046 (KB); Taebaek-si, Changchangk-dong, Geumdaebong, elev. 1300, 30 Jun 2009, Lee et al. leecs090603 (KB); Yanggugun, Yanggu-eup, Suin-ri, Mt. Samyeong, elev. 785, 21 May 2008, Paik Paik680 (KB); Pyeongchang-gun, Yongpyeongmyeon, Nodong-ri, Mt. Gyebang, elev. 790, 3 Sep 2009, Yoo \& Han 378062-0565 (KB); Chungcheongbuk-do: Jecheon-si, Dohak-dong, Mt. Solmi, elev. 240, 25 May 2013, Choi et al. J05A2075 (KB); Cheongwon-gun, Gadeok-myeon, Mt. Seondo, 25 May 1997, Jung 104 (KB); Eumseong-gun, Saenggeuk-myeon, Saeng-ri, Mt. Sureui, 27 May 2015, Lee \& Lim NAPI-P20150274 (KB); Goesan-gun, Yeonpung-myeon, Wonpung-ri, Wonpung stream, elev. 232, 12 May 2014, Kim \& Kim SHYGS120 (KB); Yeongdong-gun, Sangchon-myeon, Mt. Makgihang, elev. 250, Aug 2 2011, Kim 367164-060 (KB); Chungju-si, Salmi-myeon, Seseong-ri, Mt. Daemi, elev. 320, 29 Apr 2005, Ko 307 (KB); Danyang-gun, Gagok-myeon, Sapyeong-ri, Mt. Sobaek, elev. 471, 29 Jul 2005, Lee et al. 20050729022 (KB); Jincheon-gun, Mt. Duta, 26 Aug 2003, Paik 3311 (KB); Okcheon-gun, Iwon-myeon, Mt. Woli, elev. 100, 9 Apr 2011, Shu \& Choi 367152-005 (KB); Chungcheongnamdo: Yesan-gun, Daeheung-myeon, Sangjung-ri, Mt. Bongsu, 18 May 2014, Ahn \& Ra 10134 (KB); Cheonan-si, Gwangdeokmyeon, Mt. Gwangdeok, elev. 248, 28 Apr 2015, Cho et al. 150428106 (KB); Gongju-si, Yugu-eup, Mungeum-ri, Mt. Geumgye, elev. 352, 28 May 2015, Jeong \& Kim Geumgye150317 (KB); Taean-gun, Anmyeon-eup, Seungeonri, elev. 17, Jun 3 2010, Kim et al. MK 100096 (KB); Asansi, Baebang-myeon, Mt. Baebang, elev. 365, 30 May 2006, Ko \& Shin s.n. (KB); Seocheon-gun, Jongcheon-myeon, Doman- ri, Mt. Hwiri, 22 May 2010, Kwon $n 10063$ (KB); Dangjingun, Seongmun-myeon, Nanjido-ri, Is. Daenanji, elev. 3, 16 Sep 2014, Lim \& Seo 2014-199 (KB); Seosan-si, Unsanmyeon, Yonghyeongyegok, 30 Oct 2008, Nahm et al. SK2008034-042 (KB); Nonsan-si, Seongdong-myeon, Samho-ri, Nonsancheon, elev. 3, 16 May 2010, Oh et al. 367131-071 (KB); Boryeong-si, Misan-myeon, Mt. Wolmyeong, 18 Jul 2000, Seo et al. 265 (KB) Buyeo-gun, Mt. Huiri, 17 Jul 2000, Seo et al. 189 (KB); Geumsan-gun, Geumsan-eup, Gyejin-ri, Mt. Jinak, 25 Jul 2010, Suh \& Choi 367144-052 (KB); Jeollabuk-do: Gochang-gun, Buan-myeon, Yongsan-ri, Mt. Soyo, 24 May 2015, Ahn et al. 30049 (KB); Gunsan-si, Okdomyeon, Sinsido-ri, Is. Sinsi, 16 May 2015, Ahn et al. 50021 (KB); Jangsu-gun, Gyenam-myeon, Mt. Jangan, elev. 487, 19 Jun 2009, Ahn et al. CH43024 (KB); Imsil-gun, Mt. Seongsu, elev. 720, 17 May 2008, Choi \& Park KCH30760 (KB); Jinangun, Maryeong-myeon, Dongchon-ri, Mt. Mai, elev. 512, 20 Jun 2012, Choi \& Lee 357024-0157 (KB); Wanju-gun, Unju-myeon, Geumdang-ri, Mt. Go, elev. 180, 24 May 2008, Choi et al. SB30849 (KB); Sunchang-gun, Gurim-myeon, Geumchang-ri, Mt. Hoemun, elev. 360, 30 May 2015, Im \& Park 100084 (KB); Gimje-si, Nanbong-dong, Mt. Hwang, elev. 52, 22 May 2011, Kim \& Kwon 357014-0374 (KB); Jeongeup-si, Naejangdong, Mt. Naejang, elev. 314, 27 Jun 2007, Kim et al. 20070645 (KB); Muju-gun, Jeoksang-myeon, Sacheon-ri, Mt. Jeoksang, elev. 630, 6 Aug 2009, Kim Hyun KH3064 (KB); Namwon-si, Jusaeng-myeon, Mt. Jiri, elev.1300, 12 Jul 2007, Kim Hyun 2675 (KB); Jeonju-si, Wansan-gu,Yongbok-dong, Mt. Maebong, elev. 176, 22 May 2011, Kwon \& Kim 3570140231 (KB); Iksan-si, Geumma-myeon, Sinyong-ri, Mt. Mireuk, elev. 395, 15 May 2010, Oh et al. 364133-110 (KB); Buangun, Gyehwa-myeon, Gyehwa-ri, Mt. Gyehwa, elev. 100, 9 May 2011, Shim \& Cho 356034-085 (KB); Jeollanam-do: Kurye-gun, Mt. Chiri, around Piagol, 13 Jul 1986, Chang \& Takahasi 306 (A); Kurye-gun, Mt. Chiri-san, 16 Jul 1986, Chang \& Takahashi 357 (A); Goheung-gun, Pungyang-myeon, Andong-ri, elev. 96, 14 Jun 2011, Cho et al. 347054-0063 (KB); Hwasun-gun, Buk-myeon, Nogi-ri, Mt. Baeka (Mt. 818 m), elev. 430, 10 May 2014, Im \& Park Im90297 (KB); Naju-si, Noan-myeon, Geuman-ri, 10 May 2001, Im \& Park 013052 (KB); Gokseong-gun, Gokseong-eup, elev. 486, 20 Oct 2007, Kim Hkim3761 (KB); Gwangyang-si, Ongnyong-myeon, Mt. Baegun, elev. 1,034, 6 Jun 2009, Kim \& Jin 2530 (KB); Jangheung-gun, Gwansan-eup, Seongsan-ri, Mt. Buyong, elev. 260, 2 May 2013, Kim et al. buyong112 (KB); Jindo-gun, Uisin-myeon, Sacheon-ri, elev. 112, 4 May 2013, Kim \& Lee JJP3138 (KB); Yeosu-si, Hwayang-myeon, Anpo-ri, elev. 210, 4 Jun 2010, Kim et al. Hkim5479 (KB); Damyang-gun, Yong- 
myeon, Mt. Chuwol, 11 May 2004, Ko \& Kang 2004-8-3-185 (KB); Gurye-gun, Muncheok-myeon, Jungsan-ri, Mt. Jiri, elev. 759, 27 May 2011, Lee et al. leecs20111374 (KB); Sinan-gun, Bigeum-myeon, Is. Bigeum, Mt. Seonwang, elev. 200, 11 May 2009, Lee et al. leecs090532 (KB); Gangjin-gun, Daegumyeon, Yongun-ri, Mt. Cheontae, elev. 549, 21 May 2011, Son 346083-0025 (KB); Haenam-gun, Samsan-myeon, Gulim-ri, Mt. Duryun, elev. 200, 29 May 2014, Son \& Im Im90903 (KB); Hampyeong-gun, Hampyeong-eup, Japung-ri, Mt. Gambang, elev. 257.2, 28 May 2005, Son \& Im s.n. (KB); Yeonggwanggun, Bulgap-myeon, Moak-ri, Mt. Bulgap, elev. 378, 24 Jun 2015, Son \& Park HNHM-KB-150786 (KB); Wando-gun, Gogeum-myeon, Cheongyong-ri, Is. Gogeum, 19 May 2015, Yoo \& Lee NAPI-20150178 (KB); Boseong-gun, Joseongmyeon, Sinwol-ri, Gamabong, 12 Jun 2014, Yoon \& Jang 20140797 (KB); Muan-gun, Mt. Seungdal, Sep 4 2003, Yoon et al. 6050 (KB); Yeongam-gun, Yeongam-eup, Gaesin-ri, Mt. Wolchul, elev. 780, 27 May 2012, Yoon et al. 70673 (KB); Gyeongsangbuk-do: Sangju-si, Namjang-dong, Mt. Noeum, 4 Jun 2005, Chang \& Ryu Sky0821 (KB); Seongju-gun, Geumsumyeon, Mt. Apsok, 9 Jun 2001, Chung \& Kwon s.n. (KB); Yeongdeok-gun, Ganggu-myeon, Ganggu-ri, elev. 49, 19 May 2010, Chung \& Park 369104-0192 (KB); Chilgok-gun, Gasanmyeon, Mt. Yuhak, 6 Jun 2001, Chung \& Kwon s.n. (KB); Uiseong-gun, Oksan-myeon, Mt. Hwanghak, elev. 306, 17 Jul 2010, Hwang et al. $5 S 006$ (KB); Gunwi-gun, Gunwi-eup, Mt. Seonbang, elev. 88, 22 May 2010, Hyun et al. 368113-0055 (KB); Goryeong-gun, Mt. Mandae, 1 Jul 2000, Jeon et al. 20003921 (KB); Cheongdo-gun, Unmun-myeon, Mt. Unmun, 19 Sep 2011, Jeong et al. s.n. (KB); Uljin-gun, Geunnammyeon, Noeum-ri, Mt. Seonyu, elev. 176, 1 May 2014, Jeong et al. JKS14015 (KB); Andong-si, Pungcheon-myeon, Gisanri, Seokbang reservoir, elev. 133, 18 Jun 2014, Kim \& Kim SMY458 (KB); : Bongwha-up, 26 May 1984, Kim s.n. (A); Mt. Palgong, 20 May 1984, Kim s.n. (A); Cheongsong-gun, Budong-myeon, Hang-ri, Haewolbong, elev. 260, 21 Oct 2010, Ko \& Son HNHM-2010-2065 (KB); Mungyeong-si, Maseongmyeon, Sangnae-ri, Mt. Baekhwa, elev. 291, 18 Jun 2015, Ko \& Park HNHM-KB-150557 (KB); Yeongju-si, Punggi-eup, Jeongu-ri, Dosolbong, elev. 946, 5 Aug 2009, Ko \& Son HNHM-0999 (KB); Yeongyang-gun, Yeongyang-eup, Seoburi, 31, May 2008, Kwon \& Leen 080482 (KB); Gimcheon-si, Bongsan-myeon, Mt. Hwangak, elev. 299, 25 May 2013, Lee \& Lee 20130402 (KB); Gumi-si, Namtong-dong, Mt. Geumo, 1 Jun 2014, Lee et al. 20140161 (KB); Gyeongju-si, Yangnammyeon, Sindae-ri, Samtaebong, 10 Apr 2014, Lee \& Yoon 20140008 (KB); Pohang-si, Buk-gu, Gigye-myeon, Namgyeri, Mt. Unju, elev. 524, 24 May 2013, Lee \& Yoon 20130361
(KB); Bongwha-gun, Sukpo-myeon, 28 May 1989, March \& Meyer 122 (MO); Yeongcheon-si, Sinnyeong-myeon, Chisanri, Mt. Palgong, elev. 503, 28 Jun 2013, Park NP130068 (KB); Bonghwa-gun, Myeongho-myeon, Mt. Cheongnyang, elev. 686, 21 Apr 2013, Yoon et al. 20130131 (KB); Gyeongsan-si, Namsan-myeon, Mt. Gaya, 4 Jun 2011, Yoon \& Shin YN60006 (KB); Gyeongsangnam-do: Hadong-gun, Hwagae-myeon, Mt. Jiri, elev. 549, 27 May 2007, Chung Bulil 7 (KB); Changwonsi, Mt. Jeongbyeong, 7 May 1990, Kang 15 (KB); Changnyeong-gun, Namji-eup, Mt. Gujin, elev. 50, 20 Apr 2008, Kim \& Hong KimJH-358102-0215 (KB); Gimhae-si, Mt. Dotdae, elev. 149, 10 May 2008, Kim \& Hwang 358162-048 (KB); Hamyang-gun, Macheon-myeon, Samjeong-ri, Mt. Jiri, elev. 917, 3 Sep 2008, Kim et al. 2008-0784 (KB); Hapcheongun, Daebyeong-myeon, Hageum-ri, Mt. Hwangmae, elev. 400, 18 Jun 1998, Kim et al. 1209 (KB); Namhae-gun, Seomyeon, Namsang-ri, Mt. Mangun, elev. 650, 15 May 2010, Kim 347043-071 (KB); Yangsan-si, Habuk-myeon, Yongyeonri, Mt. Gaji, 26 Jun 2009, Kim \& Kim G407 (KB); Uiryeonggun, Chilgok-myeon, Naejo-ri, Mt. Jagul, elev. 120, 10 May 2008, Ko 2008-0730 (KB); Jinju-si, Jinseong-myeon, Mt. Jangguntaesan, elev. 251, 5 Aug 2010, Kwon \& Lee 10-1089 (KB); Geoje-si, Dongbu-myeon, Hakdong-ri, Mt. Noja, 16 Jun 2005, Lee et al. LEECS070908 (KB); Goseong-gun, Daegamyeon, Galcheon-ri, Mt. Mooryang, elev. 516, 5 Aug 2010, Lee \& Jang 10-0539 (KB); Masan-si, Naeseo-eup, Gamcheonri, Mt. Muhak, 25 Sep 2010, Lee 358151-0195 (KB); Sacheonsi, Sanam-myeon, Mt. Waryong, elev. 400, 29 May 1999, Lee et al. 1228 (KB); Tongyeong-si, Misu-dong, Mt. Hyeongeum, elev. 326, 8 May 2013, Lee \& Ahn LeeCS20130177 (KB); Miryang-si, Sannae-myeon, Mt. Cheonhwang, elev. 995, 17 Jun 2013, Son \& Park HNHM-KB-132109 (KB); Sancheonggun, Samjang-myeon, Seongnam-ri, Mt. Gamtubong, elev. 481, 11 May 2008, Sohn \& Seo E08028 (KB); Haman-gun, Gunbukmyeon, Bakgok-ri, Mt. Bangeo, elev. 77, 10 May 2008, Tae 567 (KB); Geochang-gun, Buksang-myeon, Mt. Namdeogyu, elev. 605, 13 Jul 2006, Yoo et al. 1805 (KB); Jeju-do: “in dumoris quelpaert", Jun 1907, Faurie 1564 (A); Jeju, Seogwipo-si, Namwon-eup, Wimi-ri, elev. 171, 15 Jun 2010, Kim et al. 29668 (KB); "Quelpaert in sepilus Hallaisan”, May 1909, Taquet 2806 (A); Jeju, in 1909, Taquet 2809 (A); Jeju, Mt. Halla-san, 12 Aug 1938, Uno 23577 (A, UC).

TAIWAN. Hwalien Hsien, Mt. Fong-shan, elev. 1,9002,000 m, 18 Aug 1967, Kuo et al. 6965 (MO); Ilan, Ho-Ping logroad 1790, 22 May 1985, Lu 16309 (A).

5. Neillia rubiflora D. Don, Prodr. Fl. Nepal 229, 1825.TYPE: NEPAL. Without specific locality, Wallich 697 
(holotype: K! (IDC microfiche!); isotypes: GH!, K!, NY!, P!).

Shrubs to 1-2 $\mathrm{m}$ high, with erect or spreading branches. Leaves on the flowering branches without multiple, superposed buds in their axils; blades ovate, 4-9 (-11) cm long, 3-5 (8) $\mathrm{cm}$ wide, shallowly 3 -lobed, the apex acuminate to caudate, the base cordate, the upper and lower surfaces sparsely pubescent; petioles $1.2-1.5 \mathrm{~cm}$ long; stipules lanceolate or linear, 7-9 mm long, entire, deciduous after anthesis or early deciduous. Inflorescences racemes or panicles of racemes (if panicles, 4-8 branched), 3.5-7 cm long, densely pubescent; bracts lanceolate, $4-5 \mathrm{~mm}$ long; pedicels $1-1.5 \mathrm{~mm}$ long. Hypanthium campanulate, 3-3.5 $\mathrm{mm}$ long, 4-5 $\mathrm{mm}$ wide at the widest point, densely pubescent, capitate-glandular trichomes developing after anthesis; sepals triangular, 2.5$4.2 \mathrm{~mm}$ long, apiculate at the apex, pubescent on both surfaces; petals orbicular, white; stamens 25-30, the longest filaments ca. $2 \mathrm{~mm}$ long; carpel 1, glabrous; ovules 8 per carpel. Follicles $7-8 \mathrm{~mm}$ long, with more or less erect beaks; seeds 8 per follicle, 1.6-1.8 mm long, yellowish brown.

Distribution. Bhutan, Nepal, and China in Yunnan (Fig. 6); slopes of forests, near waterfalls; 2,000-3,000 m.

Neillia rubiflora is similar to $N$. affinis and $N$. thyrsiflora but can be distinguished from the former species by having a single carpel per flower, which is glabrous or pubescent only at the apex and from the latter species by lacking multiple superposed buds in the leaf axils on flowering branches. Unlike other species of Neillia, seeds of Neillia rubiflora are yellowish brown. However, since there are only a few collections with fruiting branches, it is not clear whether the seed color can be used to delimit the species.

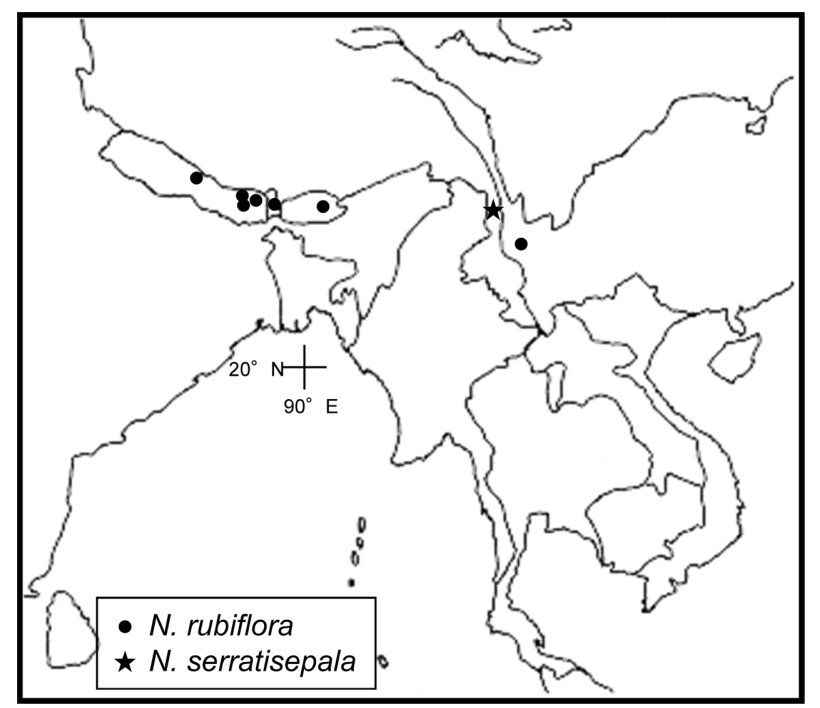

Fig. 6. The distributions of $N$. rubiflora and $N$. serratisepala.
Specimens examined. BHUTAN. Forested slopes below Namning, NW of Mongar, $27^{\circ} 19^{\prime} \mathrm{N}, 91^{\circ} 02^{\prime} \mathrm{E}$, elev. 2,300 m, 5 Jul 1979, Grierson \& Long 2492 (A); near large waterfall above Namning, NW of Mongar, $27^{\circ} 20^{\prime} \mathrm{N} 91^{\circ} 02^{\prime} \mathrm{E}$, elev. 2,600 m, 15 Jun 1979, Grierson \& Long 1936 (A).

INDIA. Sikkim, without date, Hook. f. s.n. (GH); Sirhoi, without date, Kingdon-Ward 17665 (NY); Sikkim, without date, Schneider s.n. (A).

NEPAL. Junbesi, Taksindu, elev. 8,000 ft., 22 Jul 1966, Banerji 1872 (A); Ganesh Himal, Bagmati zone, Rasuwa Distr., $28^{\circ} 14^{\prime} \mathrm{N}, 85^{\circ} 09^{\prime} \mathrm{E}, 5$ Aug 1994, Miyamoto et al. 9430092 (A); Mechi zone, Panchitar Distr., Prangbung (1,970 m) - Namle Phedi $(1,740 \mathrm{~m})$ - Namle $(2,110 \mathrm{~m})$ - Goruwale Bhanjang (Bhanduke) (2,750 m), elev. 2,600-2,800 m, 27 $10^{\prime} \mathrm{N}, 87^{\circ} 54^{\prime} \mathrm{E}-$ $27^{\circ} 05^{\prime} \mathrm{N}, 87^{\circ} 56^{\prime} \mathrm{E}, 25$ Jun 1992, Noshiro et al. 9241061 (A); Koshi zone, Sankhuwa Sabha Distr. Milke Danda, Gupha Pokhari - Angare Kharka, $27^{\circ} 20^{\prime} \mathrm{N}, 87^{\circ} 30^{\prime} \mathrm{E}, 17 \mathrm{Jul} 1991$, Ohba et al. 9110077 (A); Bhurungdi Khola, elev. 8,000 ft., 22 May 1954, Stainton et al. 5395 (A); Arun valley, Piling Khola, NE of Chyamtang, elev. 10,500 ft., 23 May 1956, Stainton 391 (A); Rambrong, Lamjung Himal, elev. 10,000 ft., 27 Jun 1954, Stainton et al. 5967 (A); Mechi zone, Taplejung Distr., $27^{\circ} 42^{\prime} \mathrm{N}$, $87^{\circ} 50^{\prime} \mathrm{E}-27^{\circ} 33^{\prime} \mathrm{N}, 87^{\circ} 47^{\prime} \mathrm{E}$, elev. 2,000 m, 2 Jun 1992, Suzuki et al. 9240479 (A).

6. Neillia serratisepala H. L. Li, J. Arnold Arbor. 25: 300, 1944.-TYPE: China. Yunnan: Shang-pa, elev. 2,000 m, a shrub $4 \mathrm{ft}$ high, flowers white, 31 Oct 1934, Tsai 59158 (holotype: A!).

Shrubs to $1.5 \mathrm{~m}$ high, with erect or spreading branches. Leaves on the flowering branches with multiple, superposed buds in their axils; blades ovate, $6-7.5 \mathrm{~cm}$ long, $2.5-4.5 \mathrm{~cm}$ wide, shallowly 3-lobed, the apex acuminate to caudate, the base truncate or cordate, the upper and lower surfaces sparsely pubescent; petioles 5-8 $\mathrm{mm}$ long; stipules ovate, 6-7 mm long, serrate, deciduous after anthesis. Inflorescences panicles of racemes, 2-3 branched, ca. $4 \mathrm{~cm}$ long, densely pubescent; bracts ovate or lanceolate, $3.8-4.8 \mathrm{~mm}$ long; pedicels $2.5-$ $5 \mathrm{~mm}$ long. Hypanthium campanulate, ca. $3.5 \mathrm{~mm}$ long, 2$2.2 \mathrm{~mm}$ wide at the widest point, densely pubescent, capitateglandular trichomes developing after anthesis; sepals triangular, 1.6-2 $\mathrm{mm}$ long, acuminate to caudate at the apex, densely pubescent on both surfaces; petals orbicular, white; stamens ca. 20, the longest filaments ca. $1.2 \mathrm{~mm}$ long; carpel 1, densely pubescent throughout the surface; ovules 3-4 per carpel.

Distribution. Fugong (Shang-pa is the old name), which is located in northwestern Yunnan in China (Fig. 6); 2,000 m. Neillia serratisepala is only known from the holotype 
specimen. This species is similar to $N$. thyrsiflora in having multiple, superposed buds in leaf axils on flowering branches, but differs in the number of ovules and the longer pedicels of the loose panicles. Cullen (1971) suggested that this species could be considered as a variety of $N$. thyrsiflora. However, because only the type specimen is available for this species, I treat it as a species (Li, 1944; Vidal, 1963; Cullen, 1971).

7. Neillia sinensis Oliv. in Hook. f., Icon Pl. 16: t. 1540, 1886; Physocarpus sinensis (Oliv.) Kuntze, Revis. Gen. Pl. 1: 218, 1891; Opulaster sinensis (Oliv.) Kuntze, Revis. Gen. Pl. 2: 949, 1891.-TYPE: China. Hupeh: District Patung Ichang, Henry $641 \& 605$ (holotype: K!). The type consists of two specimens mounted on one sheet of paper, both of which are labeled Henry $641 \& 605$. According to Vidal (1963), there is another herbarium collection at K of Henry 641, and it is the type of Lysimachia auriculata. Thus, Henry $641 \& 605$ is considered as the holotype of $N$. sinensis consisting of two branches.

Shrubs to $1.5-2 \mathrm{~m}$ high, with erect or spreading branches; branches glabrous or densely pubescent. Leaves on the flowering branches without multiple, superposed buds in their axils; blades ovate to lanceolate, 4-12 cm long, 2-7 cm wide, shallowly to more or less deeply 3-lobed, the apex acuminate to caudate, the base cordate to rounded, the upper and lower surfaces glabrous to sparsely or densely pubescent; petioles $0.5-1 \mathrm{~cm}$ long; stipules elliptic or ovate to lanceolate, 3-7 mm long, serrate or entire, deciduous after anthesis. Inflorescences racemes, 3.5-7 cm long, glabrous or densely pubescent, 1020-flowered; flowers more or less evenly distributed along the inflorescence rachis; bracts lanceolate, $8-10 \mathrm{~mm}$ long; pedicels 2.2-7 mm long. Hypanthium cylindric, 6-9 mm long, 3$4.5 \mathrm{~mm}$ wide at the widest point, glabrous to sparsely pubescent with simple unicellular or stellate trichomes at the base, capitate-glandular trichomes developing after anthesis; sepals triangular, $2.5-6 \mathrm{~mm}$ long, acuminate to caudate at the apex, pubescent on adaxial surface; petals orbicular or obovate, white or pale pink; stamens 15-25, the longest filaments ca. $2 \mathrm{~mm}$ long; carpels 1 rarely 2 , if 2 , free, pubescent only at the apex or glabrous; ovules 4-6 per carpel. Follicles 9-10 mm long, with more or less erect beaks; seeds 3-4 per follicle, $2-2.5 \mathrm{~mm}$ long, dark brown. Chromosome number: $n=9$ (Ratter and Milne, 1973).

Neillia sinensis is widely distributed in China and is morphologically very similar to $N$. thibetica in having racemes of pink flowers with cylindric hypanthia. Cullen (1971) investigated the morphological variation of the two species and concluded that they were separable by a combination of characters. According to Cullen (1971), N. sinensis is distinguished from $N$. thibetica by having glabrous hypanthia, longer hypanthia and pedicels, and fewer flowers per inflorescence. The number of ovules, which was an important character in Vidal's treatment (1964), was not useful in distinguishing the two species (Cullen, 1971). Neillia sinensis is variable in leaf size, shape, and lobation. Three varieties can be recognized as follows:

1. Flowering branches and the lower surface of leaves glabrous to sparsely pubescent …………………........... var. sinensis

1. Flowering branches and the lower surface of leaves densely pubescent.

2. Sepals 5.5-6 mm long; hypanthia sparsely stellatepubescent at the base …………………..... var. villosa

2. Sepals $2.5-4.5 \mathrm{~mm}$ long; hypanthia glabrous or sparsely pubescent with simple unicellular trichomes at the base ….......................................... var. hypomalaca

\section{7a. Neillia sinensis var. sinensis}

Neillia sinensis var. caudata Rehder in Sargent, Pl. Wilson. 1: 436, 1913; Neillia thibetica Bureau \& Franch. var. caudata (Rehder) J. E. Vidal, Adansonia 3: 164, 1963.-TYPE: China. Yunnan: Mengtze, E. Mts. elev. 6,000 ft, shrub $10 \mathrm{ft}$ with pink flowers, Henry 9669 (holotype: A!; isotypes: MO!, US).

Neillia ribesioides Rehder in Sargent, Pl. Wilson. 1: 435, 1913; Neillia sinensis var. ribesioides (Rehder) J. E. Vidal, Adansonia 3: 161, 1963.-TYPE: China. Sichuan: Pan-lan-shan, west of Kuan Hsien, elev. 2,300-3,000 m, 21 Jun and Sep 1908, Wilson 2382 (lectotype designated by Cullen, J. Arnold Arbor. 52: 152, 1971: A!).

Neillia glandulocalyx Lév., Fl. Kouy-Tchéou 348, 19141915.-TYPE: China. Guizhou: Route de Pin Fa à Tou Yun, 25 Jul 1905, Cavalerie \& Fortunat 2461 (holotype: P!; isotypes: A!, E-2 sheets!).

Neillia sinensis f. glanduligera Hemsl. ex Rehder, J. Arnold Arbor. 13: 299, 1932.-TYPE: China. Hubei: Henry 5554a (lectotype designated here: A!; isolectotypes: GH!, NY!).

Neillia fugongensis T. C. Ku, Bull. Bot. Lab. N. E. Forest. Inst., Harbin 10: 12, 1990.-TYPE: China. Yunnan: Fugong, elev. 1,700-1,800 m, 9 Sep 1982, Exped. Compl. QinghaiXizang 7162 (holotype: PE; isotype: PE!).

Neillia breviracemosa T. C. Ku, Bull. Bot. Lab. N. E. Forest. Inst., Harbin 10: 13, 1990.-TYPE: China. Yunnan: Lushui, elev. 1,950 m, 26 May 1981, Exped. Hengduanshan Inst. Bot. 029 (holotype: PE; isotype: PE!).

Branches glabrous. Blades ovate to lanceolate, $5-11 \mathrm{~cm}$ long, 3-6 cm wide, shallowly 3-lobed, the apex acuminate to 
caudate, the base cordate to rounded, the upper and lower surfaces glabrous to sparsely pubescent; stipules ovate to lanceolate, 3-7 mm long, serrate. Inflorescences glabrous to sparsely pubescent. Hypanthium 6-9 mm long, 3-4.5 mm wide at the widest point, glabrous to sparsely pubescent with simple unicellular trichomes at the base; sepals triangular, $2.5-4.5 \mathrm{~mm}$ long; ovules 4-5 per carpel.

Distribution. From Yunnan and Guangxi northward to Shaanxi, Henan, and Gansu in China (Fig. 7); river banks, wooded slopes; 400-1,800 m.

Neillia sinensis var. caudata has been characterized as having caudate to acuminate lobe apices on shallowly 3-lobed leaves. In his original description for the taxon, Rehder (1913) stated that the leaves of var. caudata resemble $N$. thyrsiflora. The holotype specimen (Henry 9669) deposited at A matches the original description. However, an isotype housed at MO has nearly unlobed ovate to lanceolate leaves with caudate apices, although the inflorescence characters are identical. In some specimens that can be ascribed to var. caudata, both types of leaves are often found in the same branch. Thus I do not consider var. caudata to be a separate taxon.

There is another Henry collection numbered 9669 at NY with his handwriting on the original label. This specimen, however, is clearly $N$. thyrsiflora. A number of collections of Neillia thyrsiflora and N. affinis var. pauciflora were also reported from the type locality of var. caudata (East Mountain in Mengtze, Yunnan). I visited East Mountain, which is also the type locality of $N$. affinis var. pauciflora, in 2000 to locate the populations of Neillia and found that the whole mountain area was deforested by gravel mining.

Neillia ribesioides was recognized based on slightly shorter

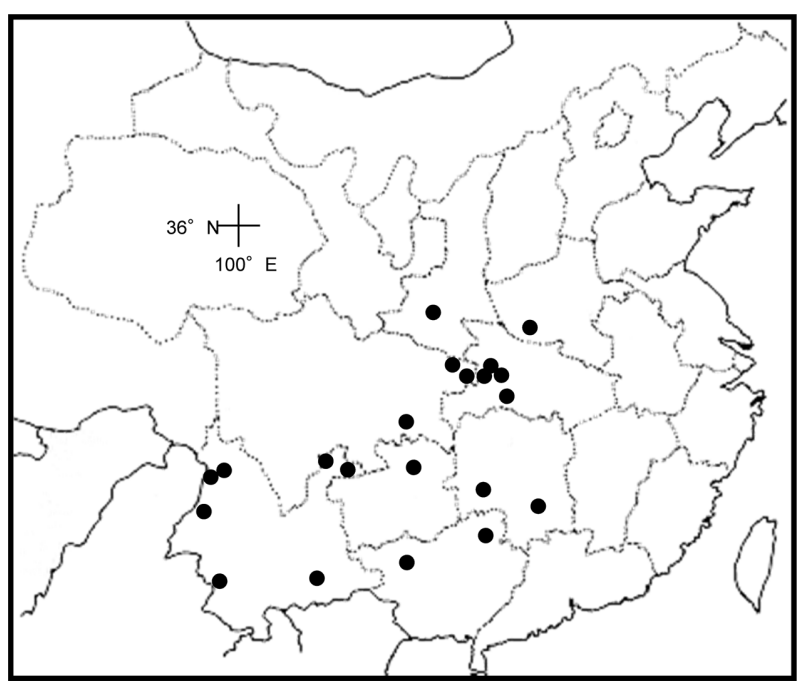

Fig. 7. The distribution of $N$. sinensis var. sinensis. hypanthia and pedicels than those of $N$. sinensis var. sinensis (Rehder, 1913; Cullen, 1971). The sizes of hypanthia and pedicels of the lectotype of Neillia ribesioides, however, are not different from those of the holotype of Neillia sinensis, and there is a considerable variation in the length of hypanthia and pedicels within an individual in many specimens. Therefore, I do not treat $N$. ribesioides as a separate taxon.

Representative specimens examined. CHINA. Gansu: Tshiluco, 18 Jun 1885, Potanin s.n. (GH); Yanchang Xian, gongjiagou (near Ganjiangtou), elev. 1,980 m, 26 Jul 1989, Yuan 1081 (MO). Guangxi: Kon Tung, Miu-shan, N Luchen, border of Guizhou, elev. 3,000 ft., 18 Jun 1928, Ching 6107 (A, NY); Hang-On-Yuen, 10 Jun 1936, Chung 81758 (A); Ling Wan District, in 1937, Lau 28719 (A); Tsuen, Ling Yun Hsien, elev. 1,000 m, 12 Apr 1933, Steward \& Cheo 172 (A, NY); Loh Hoh Tsuen, Ling Yun Hsien, elev. 1,450 m, 20 May 1933, Steward \& Cheo 450 (A, NY); Ling-chuan District, Yang-wu village \& vicinity, Ta-ling, 21-30 Jul 1937, Tsang 27882 (A). Guizhou: Jiangkoui Xian, Heiwan River on the SE side of the Fanjing-shan mountain range in the vicinity of the Ecological Station of the Guizhou Academy of Sciences, elev. 560 m, 20 Aug 1986, Sino-American Guizhou Botanical Expedition 87 (A, NY); hewon-san, Hsufeng, 4 Jul 1936, Teng 90524 (A); Top of Mt. Kyingten-shan, Tsunyi, elev. 400 m, 4 Jun 1930, Tiang 5283 (A); Guizhou Tsunyi, 4 Jun 1930, Tsiang 5283 (A); Tuyun, elev. 400 m, 11 Jul 1930, Tsiang 5744 (A). Henan: Neixiang Xian, Baotianman Nature Reserve, Xiaohongsi River, $33^{\circ} 24^{\prime} \mathrm{N}, 111^{\circ} 53^{\prime} \mathrm{E}$, elev. 1,100 m, 27 May 1994, Boufford et al. 26271 (A); Neixiang Xian, Baotianman Nature Reserve, Dahongsi River, 33⒉'N, 111 ${ }^{\circ} 54^{\prime} \mathrm{E}$, elev. 900-1,300 m, 28 May 1994, Boufford et al. 26284 (A, MO). Hubei: Patung Hsien, 14 May 1934, Chow 115 (NY); Patung Hsien, 14 May 1934, Chow 215 (A); Shenlungkai, elev. 1,800 m, in 1976, Chow 76048 (A, MO, NY); Shennongjia, elev. 980 m, 19 May 1982, Jiang \& Tao 249 (A); W of Hongriwan construction camp along trail leading towards Mt. Huanghunling, elev. 1,300 m, $31^{\circ} 30^{\prime} \mathrm{N}, 110^{\circ} 30^{\prime} \mathrm{E}, 30$ Aug 1980, Sino-Amer. Exped. 1077 (NY); Laojunshan Yaowan canyon on the $\mathrm{W}$ side of the Jiuchong River, ca. $1 \mathrm{~km} \mathrm{~S}$ of Mucheng, elev. 1,000-1,250 m, $31^{\circ} 30^{\prime} \mathrm{N}, 110^{\circ} 30^{\prime} \mathrm{E}, 31$ Aug 1980, Sino-Amer. Exped. 470 (A); trail from Quijianping along the valley of the Linzhihe River towards Laojunshan, elev. 1,500-2,700 m, 31 ${ }^{\circ} 30^{\prime} \mathrm{N}, 110^{\circ} 30^{\prime} \mathrm{E}$, 3 Sep 1980, Sino-Amer. Exped. 135 (A, NY); vicinity of Quijianping in side canyons on the E side of the Mengjia River,

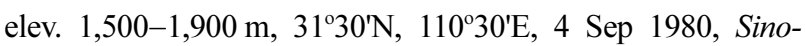
Amer. Exped. 678 (A, NY); N-San-Scian, March 1912, Silverstri 4589 (A) western Hubei, elev. 3,000-6,000 ft., 29 May 1907, Wilson 86 (A-2 sheets, GH-2 sheets); Hsing-shan- 
Hsien, elev. 4,000-6,000 ft., Jun \& Sep 1097, Wilson 189 (A-3 sheets). Hunan: Changning Hsien, Mt. Yang-shan, elev. 740 m, 13 Jul 1935, Fan \& Li 242 (A); Mt. Yun-shan prope urbem Wukang, elev. 600-1,300 m, 6 Jun 1918, Handel-Mazzetti 765 (A). Jiangxi: Mt. Yuyuang-shan, Hsieo-shui, 10 Nov 1947, Hsiung 6523 (A). Shaanxi: Tu-kia-po, 4 Jun 1897, Giraldi s.n. (A); Monte Chore-pei-san, 20 Jul 1897, Giraldi s.n. (A); Lungchow, Kuan-shan, elev. 2,000 m, 3 Jul 1922, Hers 2343 (A); Taipei-shan, in 1910, Purdom $1 \& 467$ (A). Sichuan: Wuxi Xian, Long Dong Wan, 28 May 1996, Chen et al. 960535 (MO); Nanchuan Hsien, 27 May 1928, Fang 1057 (A, NY); Cheng-kou Hsien, 13 May 1932, Fang 10315 (A, NY). Yunnan: Wei Hsi, 4 May 1933, McLaren 27D (A); Yong-shan Hsien, elev. 2,100 m, 4 Jun 1932, Tsai 50992 (A); Chen-hsiung Hsien, elev. 1,850 m, 3 Jul 1932, Tsai 52706 (A); Wei-si Hsien, Yeh-Chih, elev. 2,400 m, July 1935, Wang 67945 (A).

7b. Neillia sinensis var. hypomalaca (Rehder) Hand.-Mazz., Symb. Sin. 7: 449, 1933; Neillia hypomalaca Rehder, J. Arnold Arbor. 13: 337, 1932.-TYPE: China. Litiping range, MekongYangtze divide, east of Wei-hsi, Jun 1923, Rock 9171 (holotype: A!; photo of holotype: A!; isotypes: E!, NY!).

Neillia sinensis var. rosea Cullen, J. Arnold Arbor. 52: 153, 1971.-TYPE: China. Yunnan: Vicinity of Yunnan-sen, Maire 1120 (holotype: E!; isotype: E).

Branches densely pubescent. Blades ovate, $4-6.7 \mathrm{~cm}$ long, 2-3.5 cm wide, shallowly to more or less deeply 3-lobed, the apex acuminate to caudate, the base cordate to rounded, the upper surface moderately pubescent, the lower surface densely pubescent; stipules elliptic or ovate to lanceolate, 3-5 mm long, serrate. Inflorescences densely pubescent. Hypanthium 6$9 \mathrm{~mm}$ long, $3-4.5 \mathrm{~mm}$ wide at the widest point, glabrous to

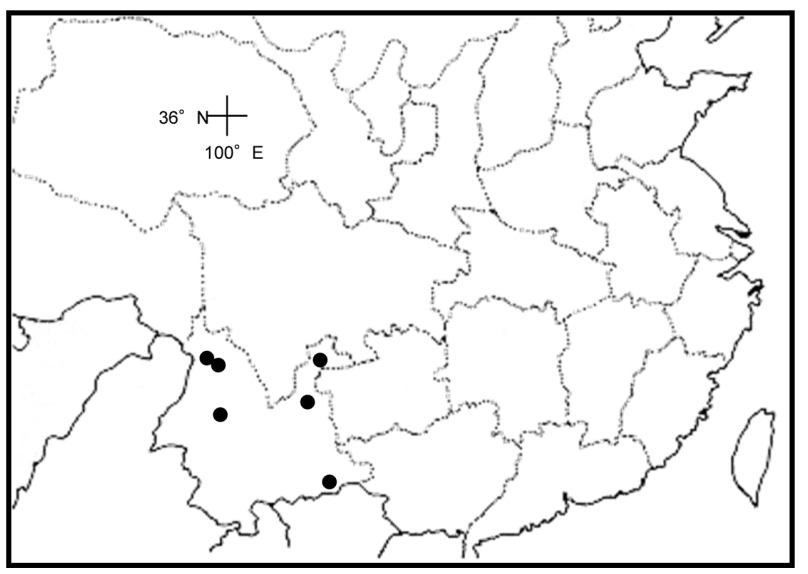

Fig. 8. The distribution of $N$. sinensis var. hypomalaca. sparsely pubescent with simple unicellular trichomes at the base; sepals triangular, $2.5-4.5 \mathrm{~mm}$ long.

Distribution. China in Yunnan (Fig. 8); 1,700-3,300 m.

Specimens examined. CHINA. Yunnan: NW Likiang, Lutien, 3 Jun 1939, Ching 20483 (A); Liu-ti-ping, 27 $12^{\prime} \mathrm{N}$, elev. 3,300 m, Jun 1917, Forrest 13883 (A); Du-long, halliers des mont, elev. 2,990 m, without date, Maire 529 (A); Tongtchouan, collines, elev. 2,990 m, without date, Maire 429 (A); Ziyang, above Yangbi, elev. 2,450 m, 10 May 1981, SinoBritish Expedition to Cangshan 456 (A); Chao-tung Hsien, elev. 1,700 m, 19 May 1932, Tsai 50992 (A); Kang-pu, Weihsi Hsien, elev. 1,932 m, Jul 1935, Wang 64159 (A).

7c. Neillia sinensis var. villosa (W. W. Sm.) Cullen, J. Arnold Arbor. 52: 153, 1971; Neillia villosa W. W. Sm., Notes Roy. Bot. Gard. Edinburgh 10: 53, 1917.-TYPE: CHINA. Yunnan: Vicinity of Yunnan-sen, in 1906, Maire 2330 (holotype: E!; isotype: E).

Branches densely pubscent. Blades lanceolate, $8-12 \mathrm{~cm}$ long, 2.8-7 cm wide, shallowly 3-lobed, the apex caudate, the base rounded, the upper and lower surfaces densely pubescent; stipules ovate, ca. $5 \mathrm{~mm}$ long, entire. Inflorescences densely pubescent with simple unicellular trichomes; pedicels 2.2$3.5 \mathrm{~mm}$ long. Hypanthium 6-7 mm long, 3-3.2 mm wide at the widest point, glabrous to sparsely pubescent with stellate trichomes at the base; sepals triangular, 5.5-6 mm long; ovules 5-6 per carpel.

Distribution. Known only from the type locality at Yunnan in China.

8. Neillia sparsiflora Rehder, J. Arnold Arbor. 1: 257, 1920.TYPE: China. Yunnan: "circa Pe-yen-tsin, Kou-ty," in 1917, Ten 462 (holotype: A!; isotype: E!).

Shrubs to $1.3 \mathrm{~m}$ high, with erect or spreading branches, pubescent with capitate-glandular trichomes. Leaves on the flowering branches without multiple, superposed buds in their axils; blades ovate, $4.5-6.5 \mathrm{~cm}$ long, $2.8-4.5 \mathrm{~cm}$ wide, shallowly 3-lobed, the apex acute to acuminate, the base cordate to rotund, the upper surface glabrous, the lower surface sparsely pubescent with capitate-glandular trichomes on the veins; petioles $1-1.5 \mathrm{~cm}$ long, pubescent with capitateglandular trichomes; stipules ovate to lanceolate, 6-7 mm long, ciliate with capitate-glandular trichomes at the margin, deciduous after anthesis. Inflorescences racemes, $3.5-4.5 \mathrm{~cm}$ long, moderately pubescent with capitate-glandular trichomes, 4-5-flowered; flowers fascicled at the apex of the 
inflorescence; bracts lanceolate, 4.6-5.1 mm long, ciliate with capitate-glandular trichomes at the margin; pedicels $3.8-5 \mathrm{~mm}$ long, pubescent with capitate-glandular trichomes. Hypanthium cylindric-campanulate to campanulate, 5.8-6.5 mm long, 4.5$5.1 \mathrm{~mm}$ wide at the widest point, glabrous or densely pubescent with capitate-glandular trichomes; sepals ca. $3.3 \mathrm{~mm}$ long, apiculate at the apex, pubescent on adaxial surface; petals obovate, white or pale pink; stamens 20-30, the longest filaments ca. $1.8 \mathrm{~mm}$ long; carpel 1 , glabrous, moderately pubescent at the apex or densely pubescent throughout the surface; ovules 7 per carpel.

Distribution. Yunnan in China (Fig. 4); mixed deciduous forests; $2,750 \mathrm{~m}$.

Neillia sparsiflora is characterized by having capitateglandular trichomes on the flowering branches, veins of the lower leaf surfaces, petioles, stipule margins, bracts, and inflorescence. It is a rare species only known from four specimens collected from Yunnan in China (Rehder, 1920; Vidal, 1964; Cullen, 1971). This species is similar to $N$. affinis and $N$. gracilis in having flowers fascicled at the inflorescence apex and campanulate hypanthia. There is polymorphism in the pubescence of the carpel. The holotype and Bai et al. 209 have glabrous carpels and the isotype has densely pubescent carpels, while Ten 462 has moderately dense pubescence at the carpel apices.

Specimens examined. CHINA. Yunnan: Dayao, under mixed forests, elev. 2,750 m, 4 Jun 1989, Bai et al. 209 (NY2 sheets); circa Pe-yen-tsin, Kou-ty, in 1918, Ten 531 (A).

9. Neillia tanakae Franch. \& Sav., Enum. Pl. Jap. 1: 121, 1873; Stephanandra tanakae (Franch. \& Sav.) Franch. \& Sav., Enum. Pl. Jap. 2: 332, 1878; Physocarpus tanakae (Franch. \& Sav.) Kuntze, Revis. Gen. Pl. 1: 219, 1891; Opulaster tanakae (Franch. \& Sav.) Kuntze, Revis. Gen. Pl. 2: 949, 1891.-TYPE: Japan. Savatier 338 (holotype: P!; isotype: K!).

Stephanandra gracilis Franch. \& Sav. Enum. Pl. Jap. 2: 333, 1878; Physocarpus gracilis (Franch. \& Sav.) Kuntze, Revis. Gen. Pl. 1: 219, 1891; Opulaster gracilis (Franch. \& Sav.) Kuntze, Revis. Gen. Pl. 2: 949, 1891.-TYPE: Japan. Savatier s.n. (holotype: P!).

Stephanandra $\times$ nakatsu-riparia Hid. Takahashi, Bull. Kanagawa Pref. Mus., Nat. Sci. 20: 17, 1991.-TYPE: Japan. Honshu: Kanagawa, Nakatsu River, Kiyokawa-mura, elev. 450 m, 21 Jun 1987, Takahashi 77096 (holotype: KPM; isotype: KPM!).

Shrubs to $2 \mathrm{~m}$ high, with erect or spreading branches. Leaves on the flowering branches without multiple, superposed buds in their axils; blades ovate, $5-10 \mathrm{~cm}$ long, $3.5-8.5 \mathrm{~cm}$ wide, shallowly $3-5$-lobed, the apex caudate, the base cordate, the middle lobes with 6-8 pairs of secondary veins, the upper surface glabrous, the lower surface sparsely pubescent; petioles $1-1.5 \mathrm{~cm}$ long; stipules ovate to lanceolate, $7-12 \mathrm{~mm}$ long, serrate or entire, persistent. Inflorescences panicles of racemes, 8-10 branched, 4-10 cm long, glabrous; bracts lanceolate, 3$5 \mathrm{~mm}$ long; pedicels $2.5-4 \mathrm{~mm}$ long. Hypanthium cupulate, $1-$ $1.5 \mathrm{~mm}$ long, $2.3-3 \mathrm{~mm}$ wide at the rim, glabrous, capitateglandular trichomes absent; sepals triangular to ovate, 1$1.8 \mathrm{~mm}$ long, apiculate at the apex, pubescent on adaxial surface; petals obovate, white; stamens 15-20, the longest filaments ca. $1 \mathrm{~mm}$ long; carpel 1, densely pubescent throughout the surface; ovules 2 per carpel. Follicles ca. $3 \mathrm{~mm}$ long, with more or less erect beaks; seeds 2 per follicle, $1.5-$ $1.7 \mathrm{~mm}$ long, dark brown. Chromosome number: $2 n=18$ (Iwatsubo and Naruhashi, 1993).

Distribution. Japan in Gumma, Kanagawa, Shizuoka, and Yamanashi prefectures (Fig. 9); rare; along streams; 200$1,300 \mathrm{~m}$.

Neillia tanakae is morphologically similar to $N$. incisa and $N$. hanceana in having cupulate hypanthia, but differs from these two species in its 15-20 stamens per flower and 3-5lobed leaves with acute or acuminate teeth. The overall leaf shape of this species resembles that of $N$. thyrsiflora. Neillia tanakae is mostly found in areas around Mt. Fuji, and it has been listed as an endangered species in Japan. Although Cullen (1971) contended that multiple, superposed buds are present in the leaf axils of flowering branches, I have not seen any specimens of $N$. tanakae with this characteristic.

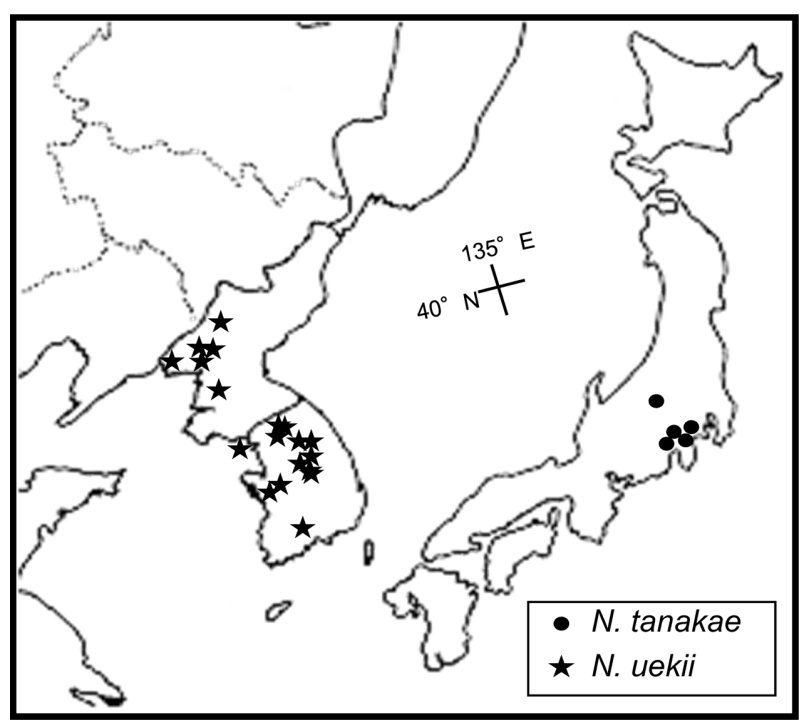

Fig. 9. The distributions of N. tanakae and N. uekii. 
Specimens examined. JAPAN. Honshu: Kanagawa Pref., Nakatsukyo, N foot of Mt Ooyama, Kiyokawa-mura, Aiko-gun, elev. 200 m, 24 Jul 1964, Fukuoka 6741 (NY, UC); Ashigarakami-gun, Kita-ashigara-mura, Tanzawa-Oyama Quasi National Park, half-way up from foot, 3 Oct 1958, Furuse s.n. (A); Sengoku hot well, Sengokubara moor, Hakone, 6 Sep 1951, Mizushima 1601 (A); Mt. Tanzawa, Kanayama-toge, elev. 800 m, 5 Aug 1963, Yamazaki 7437 (A); Shizuoka Pref., Asagirikogen, W part of Mt Fuji, on grassy field, elev. 900 m, 21 Aug 1977, Murata et al. 33874 (A); Fumoto, Asagiri Highland, Fujimiyashi, elev. 800 m, 19 Jun 1983, Togashi s.n. (A, NY, UC); Yamanashi Pref., Minami-tsuru-gun, Nishi-katsura-mura, foot of Mt Mitsutoge, 31 Aug 1957, Furuse s.n. (A); Minami-kambara, Morimachi, 10 May 1952, Ikegami 19943 (A).

10. Neillia thibetica Bureau \& Franch., J. Bot. (Morot) 5: 45, 1891.-TYPE: China. Sichuan: "environs de Ta-tsien-lou," in 1890, d'Orléans s.n. (holotype: P!).

Shrubs to 1-2.5 $\mathrm{m}$ high, with erect or spreading branches. Leaves on the flowering branches without multiple, superposed buds in their axils; blades ovate to lanceolate, $2.5-10 \mathrm{~cm}$ long, $1.8-6.5 \mathrm{~cm}$ wide, shallowly 3-lobed or deeply 3-5-lobed, the apex acute to caudate, the base cordate to rounded, the upper and lower surfaces glabrous to sparsely pubescent; petioles 3-9 mm long; stipules ovate to lanceolate, 5-9 $\mathrm{mm}$ long, serrate or entire, deciduous after anthesis. Inflorescences racemes, $3-15 \mathrm{~cm}$ long, glabrous or densely pubescent, more than 20-flowered; flowers more or less evenly distributed along the inflorescence rachis; bracts lanceolate, 6-9 mm long; pedicels $0.6-2 \mathrm{~mm}$ long. Hypanthium cylindriccampanulate, $4-5.5 \mathrm{~mm}$ long, $2.8-4 \mathrm{~mm}$ wide at the widest point, glabrous or moderately pubescent, capitate-glandular trichomes developing after anthesis; sepals triangular, $1.8-4 \mathrm{~mm}$ long, acuminate or apiculate at the apex, pubescent on adaxial surface or both surfaces; petals orbicular or obovate, pale pink; stamens ca. 20; carpel 1, rarely 2, pubescent upper half; ovules 2-7 per carpel. Follicles $8-9 \mathrm{~mm}$ long, with more or less erect beaks; seeds 3-4 per follicle, ca. $2 \mathrm{~mm}$ long, dark brown.

Neillia thibetica is morphologically very similar to $N$. sinensis, but it can be distinguished from $N$. sinensis by its shorter hypanthia, shorter pedicels, and greater number of flowers per raceme. Three varieties can be recognized as follow: 1. Hypanthia glabrous …………………........... var. duclouxii 1. Hypanthia pubescent.

2. Leaves shallowly 3-lobed; ovules 6-7 per carpel …......

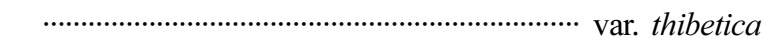

2. Leaves deeply 3-lobed; ovules 2 per carpel var. lobata

\section{0a. Neillia thibetica var. thibetica}

Neillia longiracemosa Hemsl., J. Linn. Soc., Bot. 29: 304, 1892.-TYPE: China. Sichuan: near Tachienlu, elev. 3,0004,500 m, Pratt 730 (holotype: K!; photo of holotype: A!; isotype: $\mathrm{P}$ !).

Shrubs to $2.5 \mathrm{~m}$ high. Blades ovate, 5-10 cm long, 3-6 cm wide, shallowly 3 -lobed, the apex acute to caudate, the base truncate to rounded; petioles $6-9 \mathrm{~mm}$ long; stipules ovate to lanceolate, 6-9 $\mathrm{mm}$ long, serrate or entire. Inflorescences densely pubescent, 6-15 cm long, more than 25 -flowered; pedicels $0.6-2 \mathrm{~mm}$ long. Hypanthium 4.7-5.5 mm long, 2.9$3.8 \mathrm{~mm}$ wide at the widest point, moderately pubescent with simple unicellular trichomes, capitate-glandular trichomes developing after anthesis; sepals 2.5-4 mm long, acuminate at the apex, pubescent on both surfaces; ovules 6-7 per carpel.

Distribution. China in Sichuan (Fig. 10); 1,500-3,000 m.

Specimens examined. CHINA. Sichuan: Ta Kwan, Ta Hsiang Ling, elev. 2,400 m, 7 Aug 1939, Chiao 1633 (A); Ta Hsian Ling, elev. 2,400 m, 28 May 1922, Smith 2043 (A); Mapien Hsien, 29 May 1931, Wang 23084 (A); Ta-hsiang-ling, Ching-chi Hsien, Jun 1907, Wilson 2381 (A); Mupin, elev. 5,000-7,000 ft., Jun and Sep 1908, Wilson 916 (A, GH); Tachienlu, elev. 6,000-8,000 ft., Jul and Sep 1908, Wilson 974 (A); Mupin, Oct 1910, Wilson 4220 (A); Tachienlu, elev. 8,000 ft., Oct 1910, Wilson 4220a (A); O-pien Hsien, elev. 1,500 m, 14 May 1932, Yu 740 (A); Kangding Xian, elev. 3,000 m, 2 Jun 1981, Yu \& Ku 113549 (A); Hanyuan Xian, Qingxixiang, elev. 2,000 m, 18 Jul 1989, Zhao 341 (NY).

10b. Neillia thibetica var. duclouxii Cardot ex J. E. Vidal, Adansonia 3: 163, 1963; Neillia sinensis Oliv. var. duclouxii (Cardot ex J. E. Vidal) T. T. Yu, Fl. Reipubl. Popul. Sin. 36: 91, 1974.-TYPE: China. Yunnan: "Yang Kia Ouan, prefect. Tchao Tong," 31 May 1906, Ducloux 4954 (holotype: P!).

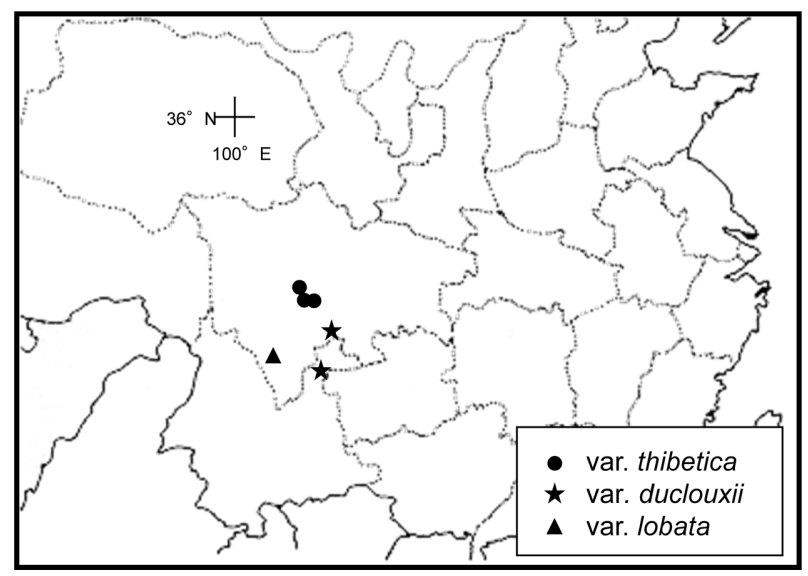

Fig. 10. The distribution of $N$. thibetica. 
Shrubs to $2.5 \mathrm{~m}$ high. Blades ovate to lanceolate, $6-9 \mathrm{~cm}$ long, 2.5-6.5 cm wide, shallowly 3-lobed, the apex acuminate to caudate, the base cordate to rounded; petioles $6-9 \mathrm{~cm}$ long; stipules lanceolate, 6-9 mm long, entire. Inflorescences 6$12 \mathrm{~cm}$ long, glabrous, more than 25-flowered; pedicels $0.6-$ $2 \mathrm{~mm}$ long. Hypanthium 4-4.5 mm long, 2.8-3.2 mm wide at the widest point, glabrous, capitate-glandular trichomes present; sepals 1.8-2 mm long, apiculate at the apex, pubescent on adaxial surface; ovules 6-7 per carpel.

Distribution. China in Sichuan and Yunnan (Fig. 10); 2,000 m.

Vidal (1963) distinguished N. thibetica var. duclouxii based on its glabrous inflorescences and hypanthia. Cullen (1971) questioned the validity of this taxon, because one of the paratypes (Wang 23084 at A) has densely pubescent inflorescences and hypanthia, suggesting that var. duclouxii does not differ from var. thibetica. Because the holotype and other type materials have glabrous inflorescences and hypanthia, as in the original description of the taxon, I recognize var. duclouxii as a separate taxon. The paratype, Wang 23084, is placed under var. thibetica in this monograph.

Specimens examined. CHINA. Yunnan: Cheou Pa Ngay, prefect. Tchao Tong, 24 May 1906, Ducloux 4611 (P); Ta Eul, pres Tchen Hiong, 3 Jul 1907, Ducloux 5215 (P); Chao-tung Hsien, elev. 2,000 m, 19 May 1932, Tsai 50929 (A).

10c. Neillia thibetica var. lobata (Rehder) T. T. Yu, Fl. Reipubl. Popul. Sin. 36: 94, 1974; Neillia longiracemosa Hemsl. var. lobata Rehder, J. Arnold Arbor. 1: 257, 1920; Neillia lobata (Rehder) J. E. Vidal, Adansonia 3: 157, 1963.TYPE: China. Sichuan: "Inter Oti et Jenyuan [Yungsin] Hsien prope Quentui [Quentin]," elev. 2,900 m, 4 Jun 1914, Schneider 3558 (holotype: A!; isotype: E!).

Shrubs to $1 \mathrm{~m}$ high. Blades ovate, $2.5-4.9 \mathrm{~cm}$ long, $1.8-$ $3.2 \mathrm{~cm}$ wide, deeply 3-lobed, the apex acute to acuminate, the base cordate; petioles 3-7 mm long; stipules lanceolate, 5$6 \mathrm{~mm}$ long, serrate. Inflorescences $3-4 \mathrm{~cm}$ long, densely pubescent, ca. 20-flowered; pedicels $0.7-1.5 \mathrm{~mm}$ long. Hypanthium 4.5-5 mm long, 3-4 mm wide at the widest point, moderately pubescent with simple unicellular trichomes, capitate-glandular trichomes developing after anthesis; sepals 2.3-4 mm long, acuminate at the apex, pubescent on both surfaces; ovules 2 per carpel.

Distribution. China in Sichuan (Fig. 10); 2,900-3,000 m.

Neillia thibetica var. lobata has been recognized as a separate species by Vidal (1963) and Cullen (1971). The leaves of this taxon are similar to those of $N$. incisa in being deeply 3-lobed.
Otherwise $N$. thibetica var. lobata is very similar to $N$. thibetica.

Specimens examined. CHINA. Sichuan: inter Tu-yung-pu et Yen-yuan Hsien, elev. 3,000 m, 12 May 1914, Schneider 4151 (A).

11. Neillia thyrsiflora D. Don., Prodr. Fl. Nepal 228, 1825; Spiraea thyrsiflora (D. Don) K. Koch, Dendrologie 1: 307, 1869; Physocarpus thyrsiflorus (D. Don) Kuntze, Revis. Gen. Pl. 1: 218, 1891; Opulaster thyrsiflorus (D. Don) Kuntze, Revis. Gen. Pl. 2: 949, 1891.-TYPE: Nepal. Without specific locality, in 1821, Wallich 698 (One that has a well-developed inflorescence at LINN designated here as lectotype (IDC microfiche!); isolectotypes: LINN (IDC microfiche!), GH!, K2 sheets!, P!). Syntype: Nepal. Hamilton s.n. (not located).

Adenilema fallax Blume, Bijdr. 1121, 1826; Neillia fallax (Blume) Blume, Mel. Bot. 1: 6, 1855 (see Steenis, Taxon 35: 272, 1986); Neillia thyrsiflora var. fallax (Blume) Kalkman, Blumea 37: 377, 1993.-TYPE: Indonesia. Java: Mt. Gede, elev. 2,000 m, Blume 477 (holotype: L!; isotype: L!).

Neillia tunkinensis J. E. Vidal, Notul. Syst. (Paris) 13: 292, 1947; Neillia thyrsiflora D. Don var. tunkinensis (J. E. Vidal) J. E. Vidal, Adansonia 3: 153, 1963.-TYPE: Vietnam. Laokay: "Col des Nuages, 25-30 km, à l'est de Phong Thô," elev. 1,500-1,600 m, Poilane 26671 (holotype: P!).

Neillia tunkinensis J. E. Vidal var. bibracteolata J. E. Vidal, Notul. Syst. (Paris) 13: 292, 1947.-TYPE: Vietnam. "environs de Chapa," elev. 1,800 m, in 1942, Pételot 6994 (holotype: P!).

Shrubs to $2.5 \mathrm{~m}$ high, with erect or spreading branches. Leaves on the flowering branches with multiple, superposed buds in their axils; blades ovate, 6-9 (-12) cm long, 4-6 (-8.5) cm wide, shallowly 3-lobed, the apex caudate or acuminate, the base cordate, the upper surface glabrous, the lower surface sparsely pubescent; petioles 1-2 cm long; stipules ovate, 6-10 mm long, serrate, deciduous after anthesis. Inflorescences panicles of racemes, 8-10-branched, 10-15 cm long, glabrous or densely pubescent; bracts lanceolate, $4.5-5 \mathrm{~mm}$ long; pedicels $0.5-$ $1.4 \mathrm{~mm}$ long. Hypanthium campanulate, $2.5-3.2 \mathrm{~mm}$ long, 2$3.2 \mathrm{~mm}$ wide at the widest point, moderately pubescent with simple unicellular trichomes, capitate-glandular trichomes developing after anthesis; sepals triangular, 3-5 mm long, acuminate to caudate at the apex, pubescent on both surfaces; petals orbicular, white; stamens 15-20, the longest filaments ca. $1 \mathrm{~mm}$ long; carpels $1-2$, if 2 free, glabrous or densely pubescent throughout the surface; ovules 6-10 per carpel. Follicles 6-6.5 mm long, with more or less erect beaks; seeds 8-10 per follicle, $1.5-1.6 \mathrm{~mm}$ long, dark brown. 


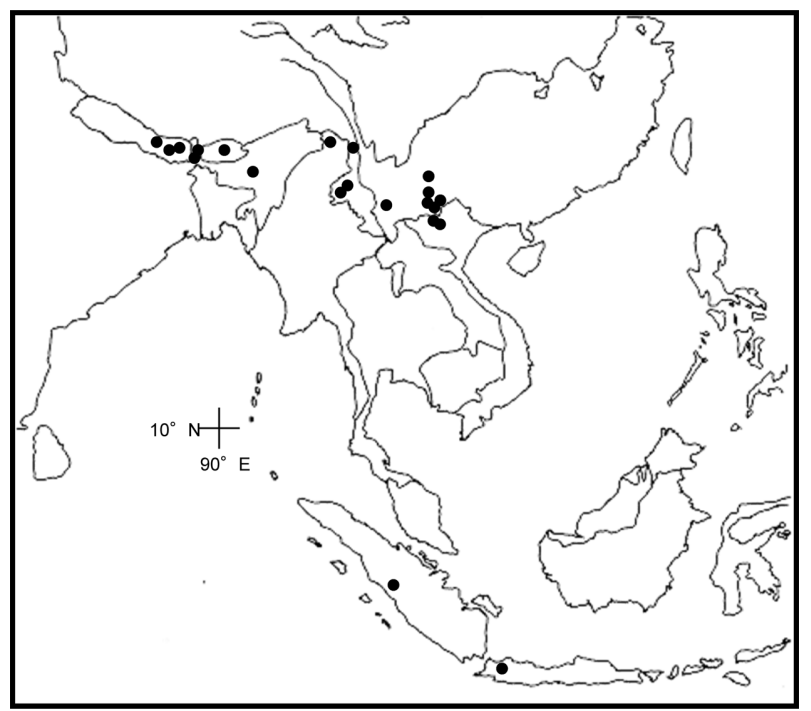

Fig. 11. The distribution of $N$. thyrsiflora.

Distribution. Nepal, India, Bhutan, eastward to Myanmar and China, southward to Indonesia (Fig. 11); mixed deciduous forests; $1,300-4,000 \mathrm{~m}$.

Neillia thyrsiflora is widely distributed from the Himalayas and southwest China to Indonesia. It is characterized by its multiple superposed buds in the leaf axils of the flowering branches, 3-lobed leaves with cordate bases, paniculate inflorescences, and flowers with white petals (Cullen 1971). Neillia fallax, described from Indonesia, is characterized by having glabrous inflorescences and glabrous carpels. Vidal (1963) recognized $N$. fallax as a separate species, while Kalkman (1993) recognized it as a variety of $N$. thyrsiflora. I do not support the segregation of $N$. fallax as a distinct taxonomic entity because there is continuous variation in the characters that have been used to distinguish it from $N$. thyrsiflora, such as leaf shape, inflorescence pubescence, number of stamens, and carpel pubescence.

Specimens examined. BHUTAN. Kamji, 19 Dec 1963, Bubbarao 273 (A).

CHINA. Guangxi: On Tak, 21 Sep 1935, Ko 55787 (A). Yunnan: Sichou Hsien, Faa-doou, elev. 1,450-1,550 m, 18 Sep 1947, Feng 11881 (A); Marlipo, Hwang-jin-i, elev. 1,1001,400 m, 13 Nov 1947, Feng 13236 (A); Marlipo, elev. 1,6002,000 m, 9 Dec 1947, Feng 13729 (A); Wen-shan-Hsien, Pyngbah, in open thickets, elev. 1,700-1,800 m, 10 Aug 1947, Feng 11044 (A); Mengtze, elev. 5,000 ft., without date, Henry 9419 (A, MO); Mengtze, S. of E Mts, elev. 4,500 ft., without date, Henry 9419A (NY); Mengtze, elev. 5,000 ft., 15 Nov without year, Henry 9419B (NY); Mengtze, without date, Henry 13653
(NY); S of Red River, without date, Henry 13653 (A); Szemao forest, elev. 4,500 ft., without date, Henry 12275 (A, MO); above Kan San Chai, W of the Mekong, en route from Pingpo to Youngchang and Tengyueh, Salween watershed, Oct 1922, Rock 7056 (A, NY); Fuchuan range, W of the Mekong-Salween divide and W of Wei-hsi, elev. 11,500 ft., Aug-Sep 1932, Rock 23375 (NY); Lu-se, elev. 1,750 m, 10 Feb 1934, Tsai 56895 (A); Pingbien Xian, in ravine, elev. 1,400 m, 15 Jun 1934, Tsai 60207 (A); Pingbien Xian, on road side, 7 Jul 1934, Tsai 62383 (A); Che-tse-lo, in forest, elev. 3,200 m, 9 Sep 1934, Tsai 58432 (A); Dzung-duei, Cham-pu-tung, mountain slope, elev. 2,000 m, Oct 1935, Wang 66940 (A); Lung-pan-la Champu-tung, meadow, elev. 3,000 m, in 1935-1936, Wang 67076 (A); Kiukiang valley (Taron) Chiengen, elev. 1,650 m, 25 Jul 1938, Yu 19409 (A); Kiukiang valley (Taron) Monting, elev. 1,350 m, 10 Sep 1938, Yu 20199 (A); Salwin-Kiukiang Divide, Newahlung, elev. 2,200 m, 8 Jul 1938, Yu 19228 (A); Mienning, Poshang, elev. 2,700 m, 9 Oct 1938, Yu 17974 (A).

INDIA. Assam (Jaintea hills), Shillong, near a waterfall between Jowan \& Jorain, 30 May 1965, Balakinshnan 42220 (MO); Katapahar, elev. 7,000 ft., 28 Dec 1937, Biswas s.n. (A, MO, NY); E Himalaya, Singmari, 5 Aug 1960, Biswas s.n. (A); Sureil, elev. 5,000 ft., 29 Aug 1912, Cave s.n. (A); E Himalaya, Ghum, elev. 7,500 ft., 4 Jul 1919, Cave s.n. (A); Shillong, elev. 6,400 ft., 9 Aug 1885, Clarke $38720 A$ (P); Mingpoo, elev. 4,000 ft., 28 Jul 1909, Cooper 556 (A); Sikkim, without date, Hook. f. s.n. (A, GH, NY); E Bengal, without date, Griffith 2115 (GH); Sinchal Lake, Darjeeling, elev. 8,000 ft., 15 Nov 1944, Relzada 19034 (A); Assam, Khasia and Jaintia Hills, elev. 5,300 ft., 12 Jun 1923, Ruse 153 (A); Sureil, 7 Sep 1921, Wilson s.n. (A-2 sheets).

INDONESIA. Java: Gunong Salak near Bogor, on path from Curugnangka to summit, $108^{\circ} \mathrm{E}, 8^{\circ} \mathrm{S}$, elev. $2,000 \mathrm{~m}, 19$ Oct 1988, Argent et al. 1058 (A); Mt. Gede, elev. 6,000 ft., 31 May 1875, Kuntze 4705 (NY-2 sheets). Sumatra: in monte ignivomo Merapi, 21 Jul 1894, Schiffner 2013 (A).

MIYANMA. N. Triangle (Hkinkum), elev. 4,000 m, $10 \mathrm{Jul}$ 1953, Kingdon-Ward 21135 (A).

NEPAL. Gurke Bandhara to Charikot, elev. 6,000 ft., 10 Oct 1960, Banerji 1210 (A); Bumlu Danda, elev. 6,500 ft., 11 Sep 1964, Banerji 1326 (A); Tamur Valley, Newa Khola, elev. 5,000 ft., 8 Aug 1956, Stainton 1292 (A).

VIETNAM. Ta Ya Ping, elev. 1,200 m, Sep 1942, Pételot $7776(\mathrm{P})$.

12. Neillia uekii Nakai, Bot. Mag. (Tokyo) 26: 3, 1912.TYPE: Korea. Without specific locality and collector's name (holotype: TI). 
Korean name: Na-do-guk-su-na-mu (나도국수나무)

Neillia millsii Dunn, Kew Bull. 1912: 108, 1912.-TYPE: Korea. [Jagang-do] Kanggye, 10 Jun 1909, Mills 107 (holotype: $\mathrm{K}$ !; isotype?: A!).

Shrubs to $2.5 \mathrm{~m}$ high, with erect or spreading branches. Leaves on the flowering branches without multiple, superposed buds in their axils; blades ovate, $5-7.7 \mathrm{~cm}$ long, $2.4-5.6 \mathrm{~cm}$ wide, shallowly 3-lobed, the apex acute to acuminate, the base cordate to rounded, the upper surface glabrous to sparsely pubescent, the lower surface sparsely to moderately pubescent; petioles 4-8 mm long; stipules elliptic or ovate, $4-8 \mathrm{~mm}$ long, serrate or entire, deciduous after anthesis. Inflorescences racemes, rarely panicles of racemes, up to 30 -flowered, 3-7 cm long, sparsely pubescent with stellate trichomes; bracts lanceolate, 4-5 mm long; pedicels $2.5-3.5 \mathrm{~mm}$ long. Hypanthium campanulate, $2.5-3 \mathrm{~mm}$ long, $2-2.2 \mathrm{~mm}$ wide at the widest point, sparsely to moderately pubescent with simple unicellular trichomes, capitate-glandular trichomes developing after anthesis; sepals triangular, 2-2.4 $\mathrm{mm}$ long, acuminate at the apex, pubescent on both surfaces; petals obovate, white; stamens ca. 20, the longest filaments ca. $1.5 \mathrm{~mm}$ long; carpel 1, glabrous; ovules 2 per carpel. Follicles $8-10 \mathrm{~mm}$ long, with more or less erect beaks; seeds 2-3 per follicle, ca. $2.8 \mathrm{~mm}$ long, dark brown.

Distribution. Korea (Fig. 9); mixed deciduous forests.

Neillia uekii is morphologically distinct in the genus in having stellate trichomes on the inflorescence rachis. The presence of stellate trichomes, common in the species of Physocarpus, is unusual in the genus Neillia. This species has been known to be endemic to Korea, occupying thickets of mountain slopes in limestome areas. Gu and Alexander (2003) reported the distribution of the species in Changdian (SE Liaoning) near the border of North Korea. I have not seen a specimen from China.

Neillia uekii and N. milsii were published in the same year of 1912. According to Vidal (1963), the name N. uekii has priority because it was published in January, 1912, whereas $N$. millsii was published in March, 1912. Cullen (1971) cited Mills collection at $\mathrm{A}$ as an isotype of $N$. milsii, but this specimen does not bear a collection number. It is unclear whether or not Mills s.n. is the duplicate of Mills 107, although both were collected on the same day.

Specimens examined. KOREA. Pyeonganbuk-do: Okkangri, 4 Jun 1914, Nakai 1776 (A); in dumosis Pyekdan, 12 Jun 1914, Nakai 1774 (A); side of stream, not common, around Mabon (Unsan), 28 Jun 1917, Wilson 8714 (A-2 sheets); Kakai [Kanggye], 5 Aug 1918, Wilson 10587 (A); Yengben, 30 Jun 1937, Smith s.n. (A); Pyeongyang-si in petrosis Pyeongyang, Jun
1901, Faurie 85 (A); Incheon: Ganghwa-gun, Gilsang-myeon, Donggeom-ri, Donggeomdo Island elev. $5 \mathrm{~m}$, Kim Donggeom12298 (KB); Gyeonggi-do: Yeoju-gun, Heungcheonmyeon, Sangbaek-ri, elev. 69 m, 30 Aug 2011, Kang sh20110830 (KB); Yeoncheon-gun, Wangjing-myeon, Gojanha-ri, elev. 49 m, 23 Jul 2008, Lee et al. 2008661061 (KB); Gangwon-do: Chuncheon-si, Namsan-myeon, Baegyang-ri, Geombong, 21 May 2004, Heo \& Choi 4416 (KB); Hongcheon-gun, Seomyeon, Mt. Palbongsan, 15 May 2009, Heo et al. 377072-1055 (KB); Jeongseon-gun, Sindong-eup, Gasa-ri, Mt. Eunbongsan, elev. 507 m, 17 Sep 2012, Jeon 378151-0391 (KB); Wonju-si, Munmak-eup, Donghwa-ri, Sajemal, 17 Jul 2013, Jeong \& Han Jul2013 (KB); Yeongwol-gun, Seo-myeon, Hutan-ri, Deulgol, elev. $197 \mathrm{~m}, 7$ Jul 2009, Jung \& Kim 0907008-1 (KB); Chungcheongbuk-do: Chugju-si, Gageum-myeon, Bonghwang recreational forest, 14 May 2009, Nam \& Lee, VS210 (KB); Danyang-gun, Maepo-eup, Pyeongdong-ri, 12 Jun 2009, Nam VS192 (KB); Goesan-gun, Yeonpung-myeon, Wonpung-ri, elev. 242 m, 12 May 2014, Kim \& Kim SHYGS41 (KB); Jecheon-si, Deoksan-myeon, Seongam-ri, elev. 166 m, 6 Jun 2013, Oh et al. J06B3004 (KB); Jincheon-gun, Mt. Dutasan, 8 Jul 2003, Paik 3312 (KB); Chungcheongnam-do: Gongju-si, Banpo-myeon, Hakbong-ri, Donghaksa, 3 Jun 2000, Ko \& Kang 1447 (KB); Yesan-gun, Daehoi-ri, Mt. Kwanyangsan, elev. $500 \mathrm{~m}$, along stream under mixed conifer forest, 25 Jul 2000, Oh 5010 (DAV); Jeollanam-do: Mt. Chirisan, 1 Aug 1934, Smith s.n. (A).

\section{Doubtful and excluded names}

The following names are excluded in this study because I have not located the type specimen and/or the original description.

Neillia densiflora T. T. Yu \& T. C. Ku, Acta Phytotax. Sin. 18: 492, 1980.-TYPE: CHINA. Xizang: Yadong Xian, elev. 2,700 m, 11 Sep 1974, Qing-Zang Exped. 74-2297 (holotype: HP?; not located).

Neillia grandiflora T. T. Yu \& T. C. Ku, Acta Phytotax Sin. 18: 493, 1980.-TYPE: CHINA. Xizang: Nyalam Xian, elev. 2,600-2,700 m, 29 Jun 1975, Qing-Zang Exped. 4452 (holotype: HP?; not located).

Neillia jinggangshanensis Z. X. Yu, Bull. Bot. Lab. N. E. Forest. Inst., Harbin 3: 150, 1983.-TYPE: China. Jiangxi: Mt. Jingganshan, elev. 400 m, 19 May 1974, without collector's name 740013 (holotype: HJA?; not located).

\section{Acknowledgments}

I am grateful to Daniel Potter, Ellen A. Dean, James A. Doyle, and two anonymous reviewers for their comments and 
suggestions on this manuscript, to Jean Shepard for managing specimen loans, and to John L. Strother and Kanchi Gandhi for sharing their comments on nomenclature. I also would like to thank the curators and staff of the herbaria listed in Materials and Methods for loans of herbarium specimens and for assistance during visits and to Suhua Shi, Yalin Peng, and Zong-Shu Yue for assistance and support with fieldwork in China. This research was supported in part by an NSF Doctoral Dissertation Improvement Grant DEB 0073041 to Daniel Potter and the author and the Eco-Innovation Project of the Ministry of Environment, Korea (Grant No. 416-111-005).

\section{Literature Cited}

Cullen, J. 1971. The genus Neillia (Rosaceae) in mainland Asia and in cultivation. Journal of the Arnold Arboretum 52: 137158.

Ohwi, J. 1965. Flora of Japan, Meyer, F. G. and E. H. Walker (eds.), Smithsonian Institution, Washington, D.C.

Oliver, D. 1886. Neillia sinensis Oliv. Plate 1540. In Hooker's Icones Plantarum. Hooker, J. D. (ed.). Williams \& Norgate, London.

Gu, C. and C. Alexander. 2003. Neillia D. Don. In Flora of China. vol. 9. Z. Y. Wu, P. H. Raven and D. Y. Hong (eds.), Science Press and Missouri Botanical Garden Press, Beijing \& St. Louis. Pp. 77-82.

Iwatsubo, Y. and N. Naruhashi. 1993. Chromosome number and karyotype of two Stephanandra species (Rosaceae). Cytologia 58: 95-98.

Kalkman, C. 1988. The phylogeny of the Rosaceae. Botanical Journal of the Linnean Society 98: 37-59.

Kuntze, O. 1891. Revisio Generum Plantarum, pars I. Arthur Felix, Leipzig

Lee, T. B. 1980. Illustrated flora of Korea. Hyangmunsa, Seoul.

Maximowicz, C. J. 1879. Adnotationes de Spiraeaceis. Trudy Imperatorskago S.-Peterburgskago Botaniceskago Sada 6: 105-261.

McNeill, J., F. R. Barrie, W. R., Buck, V. Demoulin, W. Greuter, D. L. Hawksworth, P. S. Herendeen, S. Knapp, K. Marhold, J. Prado, W. F. Prud'homme van Reine, G. F. Smith, J. H. Wiersema and N. J. Turland. 2012. International Code of Nomenclature for Algae, Fungi, and Plants (Melbourne Code). Koeltz, Königstein.

Morgan, D. R., D. E. Soltis and K. R. Robertson. 1994. Systematic and evolutionary implications of $r b c L$ sequence variation in Rosaceae. American Journal of Botany 81: 890-903.

Oh, S. 2002. A systematic study of tribe Neillieae (Rosaceae). Ph.D. dissertation submitted to University of California, Davis.

Oh, S. 2006. Neillia includes Stephanandra (Rosaceae). Novon 16: 91-95.

Oh, S. 2013. Phylogenetic analysis of PISTILLA sequences in Neillia (Rosaceae). Journal of Plant Biology 56: 145-151.

Oh, S. 2015. Taxonomy of tribe Neillieae: Physocarpus. Korean Journal of Plant Taxonomy 45: 332-352.

Oh, S. and D. Potter. 2003. Phylogenetic utility of the second intron of LEAFY in Neillia and Stephanandra (Rosaceae) and implications for the origin of Stephanandra. Molecular Phylogenetics and Evolution 29: 203-215.

Oh, S. and D. Potter. 2005. Molecular phylogenetic systematics and biogeography of tribe Neillieae (Rosaceae) using DNA sequences of cpDNA, rDNA, and LEAFY. American Journal of Botany 92: 179-192.

Oh, S., L. Chen, S. Kim, Y. Kim and H. Shin. 2010. Phylogenetic relationship of Physocarpus insularis (Rosaceae) endemic on Ulleung Island: Implications for conservation biology. Journal of Plant Biology 53: 94-105.

Potter, D., F. Gao, P. E. Bortiri, S. Oh and S. Baggett. 2002. Phylogenetic relationships in Rosaceae inferred from chloroplast $m a t K$ and $t r n L-t r n F$ nucleotide sequence data. Plant Systematics and Evolution 231: 77-89.

Potter, D., T. Eriksson, R. C. Evans, S. Oh, J. Smedmark, D. R. Morgan, M. Kerr, K. R. Robertson, M. Arsenault, T. A. Dickinson and C. Campbell. 2007. Phylogeny and classification of Rosaceae. Plant Systematics and Evolution 266: 5-43.

Rehder, A. 1913. Neillia. In Plantae Wilsonianae, part III. Sargent, C. S. (ed.). The University Press, Cambridge. Pp. 434-437.

Schulze-Menz, G. K. 1964. Rosaceae. In Engler's Syllabus der Pflanzenfamilien II, Melchior, H. (ed.), Gebrüder Borntraeger, Berlin. Pp. 209-218.

Thiers, B. [continuously updated]. Index Herbariorum: A global directory of public herbaria and associated staff. New York Botanical Garden's Virtual Herbarium. http://sweetgum.nybg.org/science/ih/.

Vidal, J. 1963. Le genre Neillia (Rosaceae). Adansonia 3: 142166.

Yu, T. and T. Ku. 1974. Physocarpus, Neillia, Stephanandra. In Flora Reipublicae Popularis Sinicae, vol. 36. Science Press, Beijing. Pp. 80-98. 